\title{
Combinations of coupled cluster, density functionals, and the random phase approximation for describing static and dynamic correlation, and van der Waals interactions
}

\author{
Alejandro J. Garza, ${ }^{a}$ Ireneusz W. Bulik, ${ }^{a}$ Ana G. Sousa Alencar, ${ }^{a}$ \\ Jianwei Sun, ${ }^{b}$ John P. Perdew ${ }^{b, c}$ and Gustavo E. Scuseria ${ }^{a, d *}$ \\ ${ }^{a}$ Department of Chemistry, Rice University, Houston, Texas 77251-1892, USA; ${ }^{b}$ Department of Physics, \\ Temple University, Philadelphia, PA 19122; ' ${ }^{c}$ Department of Chemistry, Temple University, Philadelphia, PA \\ 19122; ${ }^{d}$ Department of Physics and Astronomy, Rice University, Houston, Texas 77251-1892, USA
}

\begin{abstract}
Contrary to standard coupled cluster doubles (CCD) and Brueckner doubles (BD), singlet-paired analogues of CCD and BD (denoted here as CCD0 and BD0) do not break down when static correlation is present, but neglect substantial amounts of dynamic correlation. In fact, CCD0 and BD0 do not account for any contributions from multielectron excitations involving only same-spin electrons at all. We exploit this feature to add-without introducing double counting, self-interaction, or increase in cost - the missing correlation to these methods via meta-GGA density functionals (TPSS and SCAN). Furthermore, we improve upon these CCD0+DFT blends by invoking range separation: the short- and long-range correlations absent in CCD0/BD0 are evaluated with DFT and the direct random phase approximation (dRPA), respectively. This corrects the description of long-range van der Waals forces. Comprehensive benchmarking shows that the combinations presented here are very accurate for weakly correlated systems, while also providing a reasonable description of strongly correlated problems without resorting to symmetry breaking.
\end{abstract}

\section{INTRODUCTION}

Although coupled cluster (CC) theory is one of the pillars of quantum chemistry, commonly used CC methods (e.g., singles and doubles, CCSD) often fail in the presence of static (or strong) correlation [1, 2. These failures are connected to instabilities that appear in the random phase approximation (RPA) when Hartree-Fock (HF) becomes unstable towards symmetry breaking [2] RPAlike terms are contained in the equations of traditional coupled cluster techniques [3 5]. (RPA instabilities come in two flavors: ph-ph and pp-hh. The former is related to spin and the latter to number symmetry breaking. "Instability" in the CC context means that the solution to the CC equations results in large or complex cluster amplitudes and unbound correlation energies.) Such instabilities may be avoided by modifying the cluster operator to include only singlet-paired operators as in, e.g., pair CCD 6-11] (pCCD) and the recently proposed CCD0 method 2. However, this workaround is not a panacea: the suppression of terms in the cluster operator inevitably leads to neglecting part of the correlation. For example, CCD0 contains none of the correlation terms arising from excitations involving same-spin electron pairs which are present in CCD (see below). Despite this clear problem, pCCD and CCD0 are proper wavefunction theories: both the ansatz and its solution are rigorous approximations to solving Schrödinger equation. The main objective of the present work is to investigate ways to add the correlation that is missing in CCD0 via physically-motivated combinations of this method, and its extension using Brueckner orbitals (denoted as BD0), with techniques based on density functional theory (DFT) and the RPA.

The idea of merging techniques like CCD0 with DFT is inspired by a large number of previous works 12 45 in which multireference (MR) methods (e.g., complete active space or CAS) are mixed with density functional approximations (DFAs), in order to account for static and dynamic correlation simultaneously. The motivation for MR+DFT is that the MR ansätze typically used in quantum chemistry capture mostly (albeit not exclusively) static correlation, whereas common DFAs provide an efficient alternative to evaluate the dynamic correlation. Under this premise, one could think of approximating the exact energy as

$$
E \approx E^{\mathrm{MR}}+E_{c}^{\mathrm{DFA}}[n],
$$

where $E^{\mathrm{MR}}$ is the MR energy and $E_{c}^{\mathrm{DFA}}$ is a (dynamic) correlation functional of the density $n$. Similarly, singletpaired coupled cluster methods (CC0) can describe static correlation [2] and hence the incentive for $\mathrm{CC} 0+\mathrm{DFT}$ is analogous to that of MR+DFT. Nevertheless, despite the apparent simplicity and soundness of MR+DFT, these methods have not achieved widespread use even though concrete implementations have appeared in the literature since circa 1970 12, 13. This is largely because of three problems that plague MR+DFT:

1. Double counting. This problem arises because, in general, the MR energy contains some dynamical correlation which is also described by the DFA. A raw implementation of Eq. 1 using a standard DFA will therefore often yield too low energies and unsatisfactory results [25, 43]. Strategies that have been proposed to avoid the double counting include the use of small active spaces and reparametrized functionals [12 14; scaling the correlation energy density by factors based on the local density approximation [28 30] (LDA) or on the cusp con- 
dition for the pair density [15]; partition of the electron-electron interaction to create $\mathrm{MR}+\mathrm{DFT}$ global [39 41] or range-separated [21 25] hybrids; separation of the correlation contributions to the single-particle spectrum [31, 32]; as well partitions of the orbital space [44, 45] based on embedding schemes [46, 47]. None of these solutions is perfect: they either eliminate double counting approximately only, or eliminate it exactly but may neglect some static or dynamic correlation.

2. The symmetry dilemma. This refers to the fact that typical (e.g., Kohn-Sham (KS)) DFAs tend to break spin symmetry in strongly correlated systems [48] whereas MR methods do not. One has therefore to choose between having physically possible spin densities but unphysical energies, or improved energies but unphysical spin densities. Although one may opt on retaining the symmetries of the Hamiltonian, this choice results in massive static correlation errors for common DFAs [49. Approaches to circumvent this issue include the use of functionals that take as inputs not only the density but also the local pair density [16, 25, 28, 40, 41, or employing alternative spin densities defined by a transformation of the occupation numbers of the charge density matrix [14, 34, 35, 38, 42. The former approach is formally justified by the work of Perdew et al. 48, but can be computationally disadvantageous because computing the pair density usually requires knowledge of the two-particle density matrix, which can be expensive to evaluate [34, 35, 41. The latter approach is an ad hoc solution to the problem of computing the pair density, but suffers from ambiguity [42, 43] in the possible definitions of the alternative densities and is of course less rigorous.

3. Problems of the MR method. Typical MR techniques of quantum chemistry have serious limitations. High computational cost and the lack of size-consistency and size-extensivity are probably the most prominent of these. Of the three main problems of MR+DFT, this is the most difficult to solve as it requires the development of novel, more efficient methods for handling static correlation. Alternative MR methods that have recently been proposed for use in MR+DFT include constrainedpairing mean-field theory [38, 50] (CPMFT), projected Hartree-Fock 42, 43, 51] (PHF), pair coupled cluster doubles 6 11, 25, 41, (pCCD), and the density matrix renormalization group [23, 52] (DMRG). While they have afforded some encouraging results, none of these alternatives is flawless: CPMFT+DFT often reduces (energetically) to unrestricted $(\mathrm{U}) \mathrm{HF}+\mathrm{DFT}$ unless DFT exchange is included in the mixture 38 , (which is undesirable as it introduces self-interaction); PHF lacks size consistency and extensivity [51]; pCCD may not al- ways provide a complete description of the static correlation (e.g., when dissociating $\mathrm{N}_{2}$ it goes to higher energy limit than UHF or CPMFT [41, 53]); and DMRG still bears some of the problems of traditional MR techniques (e.g., requires definition of an active space, and size-consistency can only be guaranteed by enlarging this space). We should also mention here that attempts have also been made to add dynamic correlation to these techniques using approximations other than DFAs (see, e.g., Refs. [11, 54 61]), but, save a few exceptions [58, 59, the increase in cost is non-negligible in these approaches (and the perturbative ones can also introduce instabilities [8, 60]).

Here, we propose a different way to elude these problems of MR+DFT via CC0+DFT combinations. The double counting is avoided by using the fact that CCD0 and BD0 have only correlations involving pairs of electrons with opposite spin; we show that the missing contributions can be added in terms of a multiple of the parallel-spin correlation from a density functional. The fact that we compute solely equal-spin correlation with the DFA allows us then to avoid the typical problems caused by the symmetry dilemma without resorting to symmetry breaking. Furthermore, by using an appropriate meta-GGA (generalized gradient approximation) for the DFA, the exactness of BD0 for two-electron systems is maintained in BD0+DFT. Lastly, CCD0 and BD0 are size-consistent and size-extensive, and have polynomial cost, rather than the combinatorial cost of typical multiconfiguration techniques. Extensions incorporating RPA correlation are also derived and studied.

\section{THEORY AND METHODS}

\section{A. Singlet-Paired Coupled Cluster: CCD0 and BD0}

Like other CC methods, singlet-paired coupled cluster doubles (CCD0) starts from an exponential ansatz [2]

$$
\left|\Psi_{\mathrm{CCD} 0}\right\rangle=e^{T_{2}^{[0]}}\left|\Phi_{\mathrm{RHF}}\right\rangle,
$$

where $\left|\Phi_{\mathrm{RHF}}\right\rangle$ is a restricted Hartree-Fock reference determinant and the $T_{2}^{[0]}$ operator contains only the singletpaired component of the double excitations; i.e., if we let $i$ and $a$ be indices for occupied and virtual orbitals, respectively, $T_{2}^{[0]}$ is

$$
T_{2}^{[0]}=\frac{1}{2} \sum_{i j a b} \sigma_{i j}^{a b} P_{a b}^{\dagger} P_{i j}
$$

with

$$
\begin{aligned}
P_{i j} & =\frac{1}{\sqrt{2}}\left(c_{j \uparrow} c_{i \downarrow}+c_{i \uparrow} c_{j \downarrow}\right) \\
& =\frac{1}{\sqrt{2}}\left(c_{j \uparrow} c_{i \downarrow}-c_{j \downarrow} c_{i \uparrow}\right) .
\end{aligned}
$$


To shed light on the idea behind CCD0 (and this is helpful for understanding the justification for our CCD0+DFT combinations), it is instructive to consider standard CCD. The full double excitations cluster operator $T_{2}$ used in the latter can be expressed as 6264

$$
T_{2}=T_{2}^{[0]}+T_{2}^{[1]},
$$

where $T_{2}^{[0]}$ is defined above and

$$
T_{2}^{[1]}=\frac{1}{2} \sum_{i j a b} \pi_{i j}^{a b} \mathbf{Q}_{\mathbf{a b}}^{\dagger} \cdot \mathbf{Q}_{\mathbf{i j}}
$$

where $\mathbf{Q}_{\mathbf{i j}}$ may be written as a vector $\mathbf{Q}_{\mathbf{i j}}=$ $\left(Q_{i j}^{+}, Q_{i j}^{0}, Q_{i j}^{-}\right)^{t}$ whose components are

$$
\begin{aligned}
Q_{i j}^{+} & =c_{j \uparrow} c_{i \uparrow}, \quad Q_{i j}^{-}=c_{j \downarrow} c_{i \downarrow}, \\
Q_{i j}^{0} & =\frac{1}{\sqrt{2}}\left(c_{j \uparrow} c_{i \downarrow}-c_{i \uparrow} c_{j \downarrow}\right) \\
& =\frac{1}{\sqrt{2}}\left(c_{j \uparrow} c_{i \downarrow}+c_{j \downarrow} c_{i \uparrow}\right) .
\end{aligned}
$$

Note that $P_{i j}^{\dagger}$ acting on an empty state |\rangle gives a singlet wavefunction (hence the name of singlet-paired coupled cluster), whereas $\mathbf{Q}_{\mathrm{ij}}^{\dagger}|\rangle$ yields pure triplet components with different $m$. We see then that CCD incorporates contributions from both the singlet- and triplet-paired components of $T_{2}$. Thus, more correlation is taken into account in CCD as compared to CCD0. However, it is the combination of the singlet- and triplet-paired components what causes the failure of CCD (and other common CC approximations) in strongly correlated systems, which results in the correlation becoming too large or even complex [2]. By removing $T_{2}^{[1]}$ from $T_{2}$, CCD0 relinquishes a fraction of the correlation in exchange for safeguard against this breakdown. One may also choose to retain only the triplet-paired component in order to avoid the breakdown, but this alternative recovers less correlation than CCD0 [2].

Just as CCD can be improved by using (approximate) Brueckner orbitals in the Brueckner doubles method 6567. (BD), CCD0 has an analogous extension in singletpaired BD (BD0). Recall that the exact Brueckner orbitals are those that make the coefficients of singlysubstituted determinants in the full configuration interaction (FCI) wavefunction vanish [68. The BD0 ansatz is thus very similar to that of Eq. 2

$$
\left|\Psi_{\mathrm{BD} 0}\right\rangle=e^{T_{2}^{[0]}}\left|\Phi_{\mathrm{BD}}\right\rangle,
$$

except that the RHF reference is replaced by a determinant with approximate Brueckner orbitals $\left|\Phi_{\mathrm{BD}}\right\rangle$ which zero out the amplitudes of single substitutions in a subspace of single and double substitutions. We remark that a singlet-paired CCSD method (CCSD0) is possible too 2, but (just as it happens for BD and CCSD [69]) results from CCSD0 are not significantly different from those of BD0.

The CCD0 and BD0 methods have certain advantages over traditional MR techniques. Both algorithms scale as $\mathcal{O}\left(N^{6}\right)$; the cost of CCD0 is identical to that of CCD and the same holds for BD0 and BD. While not precisely inexpensive, it is certainly better than the combinatorial scaling of typical MR techniques and, as CC-based methods, CCD0 and BD0 may be able to take advantage of recently developed approaches 70 to decrease the cost of coupled cluster calculations. In addition, the use of exponential wavefunctions (Eqs. 2 and 9 guarantees size extensivity. Size consistency is also satisfied by the exponential ansatz provided that the reference determinant be size consistent. Lastly, it is easy to modify existing CCD and BD subroutines to implement CCD0 and BD0, respectively. Basically, most restricted CCD codes use as fundamental variables the $t_{i \uparrow j \downarrow}^{a \uparrow b \downarrow}$ amplitudes of the full double excitations cluster operator $T_{2}$, which may be expressed as 63.

$$
T_{2}=\frac{1}{2} \sum_{i j a b} t_{i \uparrow j \downarrow}^{a \uparrow b \downarrow} \sum_{\alpha \beta} c_{a \alpha}^{\dagger} c_{b \beta}^{\dagger} c_{j \beta} c_{i \alpha},
$$

and, because the singlet-paired (triplet-paired) component of $T_{2}$ is symmetric (antisymmetric) with respect to the interchange of $i$ and $j$, the $\sigma_{i j}^{a b}$ amplitudes (Eq. 3 of the singlet-paired component obey the following relations

$$
\sigma_{i j}^{a b}=\sigma_{i j}^{b a}=\sigma_{j i}^{a b}=\sigma_{j i}^{b a}=\frac{t_{i \uparrow j \downarrow}^{a \uparrow b \downarrow}+t_{i \uparrow j \downarrow}^{b \uparrow a \downarrow}}{2},
$$

which implies that we can implement CCD0 by replacing $t_{i \uparrow j \downarrow}^{a \uparrow b \downarrow}$ by $1 / 2\left(t_{i \uparrow j \downarrow}^{a \uparrow \downarrow}+t_{i \uparrow j \downarrow}^{b \uparrow a \downarrow}\right)$ at each iteration in a CCD code. Analogous remarks apply for the implementation of BD0 starting from BD subroutines.

The above discussion regarding $\mathrm{CCD} 0$ and BD0 suffices for the purposes of this paper. The interested reader may found further details about singlet-paired coupled cluster in Ref. [2].

\section{B. Adding DFT Correlation to CCo Without Double Counting: CCO+DFT}

To combine CCD0 and BD0 with density functionals while avoiding double counting, we first note that the CCD0 wavefunction can be expanded as

$$
\begin{aligned}
e^{T_{2}^{[0]}}\left|\Phi_{\mathrm{RHF}}\right\rangle & =\left|\Phi_{\mathrm{RHF}}\right\rangle+T_{2}^{[0]}\left|\Phi_{\mathrm{RHF}}\right\rangle+\frac{T_{2}^{[0]} T_{2}^{[0]}}{2 !}\left|\Phi_{\mathrm{RHF}}\right\rangle+\cdots \\
& =\left|\Phi_{\mathrm{RHF}}\right\rangle+\sum_{i j a b} \sigma_{i j}^{a b} c_{a \uparrow}^{\dagger} c_{b \downarrow}^{\dagger} c_{j \downarrow} c_{i \uparrow}\left|\Phi_{\mathrm{RHF}}\right\rangle+\cdots,
\end{aligned}
$$

and that the CCD0 correlation energy is

$$
E_{c}^{\mathrm{CCD} 0}=\sum_{i j a b} \sigma_{i j}^{a b} v_{i \uparrow j \downarrow}^{a \uparrow \uparrow \downarrow},
$$


where $v_{i j}^{a b}=\langle i j \mid a b\rangle$ is a two-electron integral in the Dirac notation. It is clear from these expressions that CCD0 neglects contributions to the correlation from equal-spin excitations: only integrals involving pairs of oppositespin electrons in occupied or virtual orbitals are weighted in the correlation energy. The same applies to BD0 except, of course, that the orbitals $i, j, a$, and $b$ would be (approximate) Brueckner, rather than RHF. One can thus think of adding equal spin correlation to CCD0 using a density functional; the correlation of the parallel-spin density given by a functional contains, in principle, contributions from all excitations involving same-spin electrons only. Hence, we may, in the spirit of Eq. 1. write

$$
E_{c}^{\mathrm{CCD} 0+\mathrm{pDFT}}=E_{c}^{\mathrm{CCD} 0}+E_{c \uparrow \uparrow}^{\mathrm{DFA}}\left[n_{\uparrow}, n_{\downarrow}\right]+E_{c \downarrow \downarrow}^{\mathrm{DFA}}\left[n_{\uparrow}, n_{\downarrow}\right],
$$

where the "p" in pDFT is for "parallel-spin" and $E_{c \alpha \alpha}^{\mathrm{DFA}}$ is the correlation of the spin- $\alpha$ density computed by a DFA. To evaluate $E_{c \alpha \alpha}^{\mathrm{DFA}}$, we can use the exchange-like spin resolution of the correlation by Stoll et al. 71]

$E_{c \uparrow \uparrow}^{\mathrm{DFA}}\left[n_{\uparrow}, n_{\downarrow}\right]+E_{c \downarrow \downarrow}^{\mathrm{DFA}}\left[n_{\uparrow}, n_{\downarrow}\right]=E_{c}^{\mathrm{DFA}}\left[n_{\uparrow}, 0\right]+E_{c}^{\mathrm{DFA}}\left[0, n_{\downarrow}\right]$.

Also, because CCD0 and BD0 do not break spin symmetry and we will be working only with closed shells, the total CCD0+pDFT energy simplifies to

$$
E_{\text {total }}^{\mathrm{CCD} 0+\mathrm{pDFT}}=E^{\mathrm{CCD} 0}+2 E_{c}^{\mathrm{DFA}}\left[n_{\uparrow}, 0\right],
$$

and likewise for BD0.

Now, looking back at the triplet-paired component of $T_{2}$ that is excluded in CCD0 (Eqs. 6. 7, and 8), we realize that, apart from the same-spin correlation, CCD0 misses opposite-spin contributions from the $m=0$ component of $T_{2}^{[1]}\left(Q_{i j}^{0}\right.$ in Eq. 8). However, for a closed shell, the $m=+1, m=0$, and $m=-1$ channels of $T_{2}^{[1]}$ contribute all equally to the energy. In other words, the spin-up correlation associated with the $m=+1$ element of $T_{2}^{[1]}$ is, by symmetry, identical to the opposite-spin correlation of the $m=0$ part. We may therefore incorporate the opposite-spin energy that is missing in CCD0+pDFT by adding $E_{c}^{\mathrm{DFA}}\left[n_{\uparrow}, 0\right]$ once more to the total energy

$$
E_{\text {total }}^{\mathrm{CCD} 0+\mathrm{tDFT}}=E^{\mathrm{CCD} 0}+3 E_{c}^{\mathrm{DFA}}\left[n_{\uparrow}, 0\right],
$$

where the "t" in tDFT indicates that the full contributions from the triplet-paired component of $T_{2}$ are being taken into account. Again, the above analysis applies to BD0 too.

In this work, we employ Eqs. 16 and 17 in a non-selfconsistent manner: after a self-consistent CCD0 (BD0) calculation, the equal spin correlation is obtained in a single-shot evaluation of a DFA using the densities from the reference RHF (Brueckner) determinant. This is a reasonable assumption because, when adding DFA dynamic correlation to $\mathrm{MR}$ wavefunctions, the error in the approximate functional is often larger than the error due to lack of self-consistency 72. We benchmark both $\mathrm{CC} 0+\mathrm{pDFT}$ and $\mathrm{CC} 0+\mathrm{tDFT}$ with $\mathrm{CC} 0$ referring to both CCD0 and BD0. The motivation for testing both $\mathrm{CC} 0+\mathrm{pDFT}$ and $\mathrm{CC} 0+\mathrm{tDFT}$ is that if the $\sigma_{i j}^{a b}$ amplitudes are close to those of the full configurationinteraction (FCI), and the same-spin DFA correlation is fairly accurate, then we would anticipate $\mathrm{CC} 0+\mathrm{tDFT}$ to outperform $\mathrm{CC} 0+\mathrm{pDFT}$. However, if it is not the case that the $\sigma_{i j}^{a b}$ amplitudes of $\mathrm{CC} 0$ are similar to the exact ones, then $\mathrm{CC} 0+\mathrm{tDFT}$ may overcorrelate and not necessarily be better than $\mathrm{CC} 0+\mathrm{pDFT}$.

\section{Eluding the Symmetry Dilemma and Selecting an Adequate Density Functional}

So far we have identified the advantages of $\mathrm{CC} 0$ over traditional MR techniques, and the derivation of $\mathrm{CC} 0+\mathrm{DFT}$ above explains how double counting is avoided; we are just left to deal with the symmetry dilemma. To illustrate how this issue can be avoided, consider the paradigm for static correlation in quantum chemistry: the $\mathrm{H}_{2}$ singlet molecule at the dissociation limit 49. For the exact, symmetry-adapted, wavefunction, $n_{\uparrow}$ and $n_{\downarrow}$ are distributed equally in each atom, but the probability of finding both electrons at the same atom is zero. That is, the correlation is purely static and there is no dynamic correlation because the electrons are never close to each other. However, with symmetry-adapted densities, common DFAs yield a nonzero $E_{c}^{\text {DFA }}$ that is caused (largely or completely) by the fact that semilocal approximations depending on the density and its gradients "see" the densities of two different electrons on top of each other; they do not know that the local pair density is zero everywhere. This problem is normally alleviated by breaking spin symmetry in a way that localizes an electron of a certain spin around a specific nucleusso that the functional won't see the densities of different electrons overlapping - or by introducing functionals that depend not only on the density, but also on the local pair density [48].

Because we evaluate the residual correlation in terms of parallel-spin correlation only (see Eqs. 16 and 17), the spurious opposite-spin correlation due to the use of symmetry-adapted densities when describing the dissociation limit of $\mathrm{H}_{2}$ (and stretched bonds in general) disappears in CC0+DFT. Therefore, there is no need for symmetry breaking or using the local pair density. Nonetheless, we should still consider the problem of selfinteraction in approximate functionals, which produces artificial self-correlation and is present in the local density approximation (LDA) and generalized gradient approximations (GGAs). One can get rid of this issue, at least in the case of two-electron systems, by using metaGGA functionals: DFAs that depend on the density, its gradient, and the kinetic energy density. We have therefore chosen to test here combinations of $\mathrm{CC} 0$ with two meta-GGAs: the Tao-Perdew-Staroverov-Scuseria 73. (TPSS) functional, and the recently developed strongly constrained and appropriately normed (SCAN) func- 
tional of Sun et al. [74. These functionals were selected based on the facts that (1) they are nonempirical; (2) they are free of one-electron self-interaction error, so that they yield no same-spin correlation for two-electron singlets and both BD0+TPSS and BD0+SCAN will be exact for these systems; and (3) that despite these similarities, TPSS and SCAN are quite different because they were designed based on different paradigms. Specifically, TPSS respects the paradigms of describing correctly oneor two-electron densities and slowly varying densities 73 , whereas SCAN obeys all the 17 known exact constrains that a meta-GGA can obey, and is exact or near exact for a set of rare-gas atoms and certain nonbonded interactions [74] (the so-called "appropriate norms").

\section{Improving the Spin Resolution of the SCAN correlation}

In Section 2.2 we used the spin resolution of $E_{c}^{\mathrm{DFA}}$ by Stoll et al. [71] $\left(E_{c \uparrow \uparrow}^{\mathrm{DFA}}\left[n_{\uparrow}, n_{\downarrow}\right]=E_{c}^{\mathrm{DFA}}\left[n_{\uparrow}, 0\right]\right)$ to extract the parallel-spin correlation. However, this decomposition is correct only for fully spin-polarized densities and in the high-density limit of the uniform electron gas 75 . In other regimes of the uniform gas, it exaggerates the equal-spin correlation. It is possible, however, because of how the correlation is composed in SCAN, to formulate a better educated guess for the spin resolution of this functional.

The dependence on the kinetic energy density, $\tau(r)=$ $\sum_{i}^{\text {occ }}(1 / 2)\left|\nabla \psi_{i}(r)\right|^{2}$, is introduced in SCAN via the dimensionless variable $\alpha$ [74]:

$$
\alpha=\left(\tau-\tau^{W}\right) / \tau^{\text {unif }}>0,
$$

where $\tau^{W}=|\nabla n|^{2} / 8 n$ is the single-orbital limit of $\tau$, and $\tau^{\text {unif }}$ is the uniform density limit. SCAN constructs the correlation energy density $\varepsilon_{c}$ by interpolation and extrapolation from the $\alpha=0\left(\varepsilon_{c}^{0}\right)$ and $\alpha=1\left(\varepsilon_{c}^{1}\right)$ limits. It is therefore natural to build its spin resolution in the same way:

$$
\varepsilon_{c}^{\uparrow \uparrow}=\varepsilon_{c}^{1 \uparrow \uparrow}+f_{c}(\alpha)\left[\varepsilon_{c}^{0 \uparrow \uparrow}-\varepsilon_{c}^{1 \uparrow \uparrow}\right],
$$

where $f_{c}(\alpha)$ (which is given in the Supporting Information of Ref. [74]) satisfies $f_{c}(0)=1, f_{c}(1)=0$, and $f_{c}(\infty)=-0.70$, and likewise for the spin-down component. We note that $\varepsilon_{c}^{0 \uparrow \uparrow}=\varepsilon_{c}^{0 \downarrow \downarrow}=0$, since there is no parallel-spin correlation for two electrons in the same spatial orbital. Thus, the fraction of same-spin correlation in SCAN depends only on the fraction of same-spin correlation in the uniform density limit, $\varepsilon_{c}^{1 \uparrow \uparrow}+\varepsilon_{c}^{1 \downarrow \downarrow}=$ $\left(1-F_{\uparrow \downarrow}\right) \varepsilon_{c}^{1}$. The total parallel-spin correlation energy density thus becomes

$$
\varepsilon_{c}^{\uparrow \uparrow}+\varepsilon_{c}^{\downarrow \downarrow}=\left(1-f_{c}(\alpha)\right)\left(1-F_{\uparrow \downarrow}\right) \varepsilon_{c}^{1} .
$$

All of the terms in the above equation are already determined in the original SCAN functional except for $F_{\uparrow \downarrow}$, the fraction of opposite-spin correlation density in the uniform density limit. Gori-Giorgi and Perdew [75] have worked out the spin resolution for the uniform electron gas: they determined fractions $F_{\sigma \sigma^{\prime}}$ such that $\varepsilon_{c}^{\sigma \sigma^{\prime}}=$ $\varepsilon_{c} F_{\sigma \sigma^{\prime}}$ in terms of the local Wigner-Seitz radius $r_{s}=$ $(4 \pi n / 3)^{-1 / 3}$ and the spin polarization $\zeta=\left(n_{\uparrow}-n_{\downarrow}\right) / n$. Therefore, we calculate $F_{\uparrow \downarrow}\left(r_{s}, \zeta\right)$ using Equation 9 of Ref. 75, which is an interpolation between exact results for the high $\left(r_{s} \rightarrow 0\right)$ and low $\left(r_{s} \rightarrow \infty\right)$ density limits that agrees with available quantum Monte Carlo data 76 in the range $0.8 \leq r_{s} \leq 10$ with $\zeta=0$.

Note that integrating Eq. 20 yields all the SCAN correlation that needs to be added in $\mathrm{CC} 0+\mathrm{pDFT}$; for CC0+tDFT, this energy should be multiplied by $3 / 2$ (see Section 2.2). Also, Eq. 20 gives no correlation for twoelectron singlets because, for these systems, $\alpha=0$ and $f_{c}(0)=1$. Thus, BD0+SCAN remains exact for twoelectron systems when the parallel-spin correlation is resolved using Eq. 20.

\section{E. Modeling the Long-Range Correlation with the Random Phase Approximation}

Adding residual correlation to $\mathrm{CC} 0$ via semilocal DFAs has the following pitfall: Semilocal functionals can't capture the long-range part of the correlation in van der Waals interactions [77, 78]. Therefore, the description of these forces in $\mathrm{CC} 0+\mathrm{DFT}$ is essentially the same as that of $\mathrm{CC} 0$. Because van der Waals forces are very sensitive to dynamic correlation, and $\mathrm{CC} 0$ misses a substantial part of it (the triplet pairing channel, to be precise), $\mathrm{CC} 0+\mathrm{DFT}$ may be inadequate for simulating this sort of interactions.

This problem can be solved by the technique of rangeseparation [79 84]: The interelectron Coulomb operator $r_{12}^{-1}$ is separated into a short-range (SR) component and its long-range (LR) complement

$$
\frac{1}{r_{12}}=\underbrace{\frac{1-\operatorname{erf}\left(\mu r_{12}\right)}{r_{12}}}_{\mathrm{SR}}+\underbrace{\frac{\operatorname{erf}\left(\mu r_{12}\right)}{r_{12}}}_{\mathrm{LR}},
$$

where erf is the error function and $\mu$ a parameter defining the range separation. (Other partitions of $r_{12}^{-1}$ are possible, but the above choice is most convenient as it has been used to parametrize screened functionals and also facilitates the evaluation of two-electron integrals.) We can then evaluate the DFA correlation with the SR interaction only; the LR complement should be computed with an approximation capable of describing the longrange part of the van der Waals forces in a way that does not add double counting to $\mathrm{CC} 0$ (and, desirably, that won't increase the cost). As we shall explain shortly, a suitable approach that satisfies all of these requirements consists in applying the direct random phase approximation (dRPA) in a manner similar to that by Janesko et al. 85. 
For the sake of clarity, let us consider how the RPA models correlation. The RPA requires the solution of an eigenvalue equation of the form [3, 85, 86]

$$
\left(\begin{array}{cc}
\mathbf{A} & \mathbf{B} \\
-\mathbf{B} & -\mathbf{A}
\end{array}\right)\left(\begin{array}{cc}
\mathbf{X} & \mathbf{Y} \\
\mathbf{Y} & \mathbf{X}
\end{array}\right)=\left(\begin{array}{cc}
\mathbf{X} & \mathbf{Y} \\
\mathbf{Y} & \mathbf{X}
\end{array}\right)\left(\begin{array}{cc}
\boldsymbol{\omega} & 0 \\
0 & -\boldsymbol{\omega}
\end{array}\right)
$$

where $\mathbf{A}, \mathbf{B}, \mathbf{X}$, and $\mathbf{Y}$ are all of dimension $o v \times o v$, with $o$ and $v$ being the number of occupied and virtual spinorbitals, respectively. In the full RPA, the elements of $\mathbf{A}$ and $\mathbf{B}$ are

$$
\begin{gathered}
A_{i a, j b}=\left(\epsilon_{a}-\epsilon_{i}\right) \delta_{i j} \delta_{a b}+\langle i b \| a j\rangle, \\
B_{i a, j b}=\langle i j \| a b\rangle .
\end{gathered}
$$

Equations 22 24 are used for the calculation of excited states. To obtain the ground state correlation energy, one notes that the Tamm-Dancoff approximation (also known as configuration interaction singles) also computes excited states with Eq. 22 but setting $\mathbf{B}=0$ (i.e., it solves for $\mathbf{A Z}=\mathbf{Z} \boldsymbol{\nu})$. While this approximation contains only excitation operators, the RPA also includes de-excitation operators that can be thought of as correlating the ground state. Thus, the RPA correlation energy for the ground state is normally written as [3, 91

$$
E_{c}^{\mathrm{RPA}}=\frac{1}{2} \operatorname{Tr}(\boldsymbol{\omega}-\mathbf{A}) .
$$

In the dRPA, the correlation is given by the same expression, except the the exchange contributions are neglected [3, 85, 86] (i.e., $\langle p q \| \mid r s\rangle$ is replaced by $\langle p q \mid r s\rangle$ in Eqs. 23 and 24). The dRPA has been most useful for incorporating correlation in methods that already contain exchange (see, e.g., Refs. [3, 85, 87] and references therein). Direct RPA also has the enormous advantage that the correlation is guaranteed to be real if the orbitals obey the aufbau principle, which is not true for the full RPA [88. As a matter of fact, the full RPA correlation becomes complex in the presence of an RHF instability [89, 90] (negative eigenvalue in the Hessian). The dRPA does not suffer any of the instability problems discussed above for full RPA or CCD because of the neglect of exchange. This minimalist description of the RPA correlation is sufficient for our purposes here; the interested reader is referred to reviews on the subject 86, 91 for further details.

From this explanation, and the analysis in Section 2.2, it is straightforward to see how long-range dRPA correlation may be added to $\mathrm{CC} 0$ without double counting. The correlation is still expressed by Eq. 25. only the elements of $\mathbf{A}$ and $\mathbf{B}$ need to be altered:

$$
\begin{gathered}
A_{i a, j b}=\left(\epsilon_{a}-\epsilon_{i}\right) \delta_{i j} \delta_{a b}+\left\langle i b\left|v_{e e}^{\mathrm{lr}}\right| a j\right\rangle, \\
B_{i a, j b}=\delta_{\sigma_{i} \sigma_{j}}\left\langle i j\left|v_{e e}^{\mathrm{lr}}\right| a b\right\rangle,
\end{gathered}
$$

where $\delta_{\sigma_{i} \sigma_{j}}=1$ if the spin functions of the spinorbitals $\chi_{i}(\mathbf{x})$ and $\chi_{j}(\mathbf{x})$ are identical and $\delta_{\sigma_{i} \sigma_{j}}=0$ otherwise, and $\left\langle i j\left|v_{e e}^{\mathrm{lr}}\right| a b\right\rangle$ indicates that the two-electron integral be evaluated with the long-range interaction

$$
\begin{aligned}
&\left\langle i j\left|v_{e e}^{\mathrm{lr}}\right| a b\right\rangle=\int d \mathbf{x}_{1} d \mathbf{x}_{2} \chi_{i}^{*}\left(\mathbf{x}_{1}\right) \chi_{j}^{*}\left(\mathbf{x}_{2}\right) \frac{\operatorname{erf}\left(\mu r_{12}\right)}{r_{12}} \times \\
& \chi_{a}\left(\mathbf{x}_{1}\right) \chi_{b}\left(\mathbf{x}_{2}\right) .
\end{aligned}
$$

By calculating the dRPA correlation with the $\mathbf{A}$ and $\mathbf{B}$ matrices as defined in Eqs. 26 and 27, only parallel spin correlation that does not overlap with the $\mathrm{CC} 0$ correlation is obtained. This is the same strategy that we utilized in Section 2.2 to avoid the double counting between DFT and CC0. Therefore, the same considerations discussed in Section 2.2 apply; one can add to CC0 only the parallel-spin correlation, or the full triplet-paired component contributions by multiplying the total equal-spin correlation by $3 / 2$.

The RPA correlation describes correctly dispersion and van der Waals interactions [92, 93, and is exact for longrange correlations 94. In addition, Scuseria et al. [3] have shown that solving the RPA eigenvalue problem of Eq. 22 is equivalent to solving for $\mathbf{T}=\mathbf{Y X}^{-1}$ in a Riccati CCD equation

$$
\mathbf{B}+\mathbf{A T}+\mathbf{T A}+\mathbf{T B T}=0,
$$

and that $E_{c}^{\mathrm{RPA}}$ in Eq. 25 can also be expressed as

$$
E_{c}^{\mathrm{RPA}}=\frac{1}{2} \operatorname{Tr}(\mathbf{B T}) .
$$

Using the Cholesky decomposition of $\mathbf{A}$ and $\mathbf{B}$, Equation 29 can be solved in $\mathcal{O}\left(N^{4}\right)$ computational effort [3, 85, where $N$ is the number of basis functions. This does not exceed the cost of CCD0. Hence, the dRPA correlation fulfills all of the requirements that we were looking for: it can be added to CCD0 without double counting or increase in scaling, and it describes properly long-range interactions. As we do for $E_{c}^{\mathrm{DFA}}$, the dRPA correlation is obtained from a single-shot, post-SCF, calculation using the RHF or BD orbitals and added to the CC0 energy.

As mentioned above, $E_{c}^{\mathrm{DFA}}$ needs to be evaluated with the SR interaction only, in order to avoid double counting with the dRPA correlation. To the best of our knowledge, there are no parametrizations for the short-range correlation of TPSS or SCAN. However, codes for the range-separated parametrization of the LDA are available [95. Therefore, we use an approximate local scaling to estimate the SR meta-GGA same-spin correlation

$$
E_{c \uparrow \uparrow}^{\mathrm{sr}, \mu}[n]=\int n_{\uparrow}(r) \frac{\epsilon_{c, \mu}^{\mathrm{sr}-\mathrm{LDA}}\left(n_{\uparrow}, 0\right)}{\epsilon_{c}^{\mathrm{LDA}}\left(n_{\uparrow}, 0\right)} \epsilon_{c \uparrow \uparrow}^{\mathrm{MGGA}}(n, \nabla n, \tau) d^{3} r,
$$

where $\epsilon_{c, \mu}^{\mathrm{sr}-\mathrm{DFA}}$ and $\epsilon_{c}^{\mathrm{DFA}}$ are short- and full-range DFA correlation energy densities, respectively, taking spin densities as inputs. A similar LDA-based scaling has been shown to produce reasonable results in our recent work on range-separated hybrids of pCCD and density 
TABLE I: Summary of the CC0+DFT methods tested here. The notation, closed-shell energy formulas, and relevant equations are given; $\mathrm{CC} 0$ can refer to CCD0 or BD0 and the densities are from the RHF or Brueckner reference determinants, respectively; the "p" in pDFT is for parallel spin; the "t" in tDFT is for triplet-pairing component; $E_{c \uparrow \uparrow}^{\mathrm{sr} \uparrow \mathrm{rSAN}}$ is the spin-up short-range SCAN correlation using the spin resolution of Section $2.4 ; E_{c \uparrow \uparrow}^{\mathrm{lr}-\mathrm{dRPA}}$ is the spin-up long-range dRPA correlation; and the rest of the notation is given in the text.

\begin{tabular}{|c|c|c|}
\hline Method & Energy Formula & Relevant Eqs. \\
\hline$\overline{\mathrm{CC} 0+E_{c}^{\mathrm{DFT}}}$ & $E^{\mathrm{CC} 0}+E_{c}^{\mathrm{DFA}}\left[n_{\uparrow}, n_{\downarrow}\right]$ & See Sec. 2.6. \\
\hline $\mathrm{CC} 0+\mathrm{pDFT}$ & $E^{\mathrm{CC} 0}+2 E_{c}^{\mathrm{DFA}}\left[n_{\uparrow}, 0\right]$ & 14,15 \\
\hline $\mathrm{CC} 0+\mathrm{tDFT}$ & $E^{\mathrm{CC} 0}+3 E_{c}^{\mathrm{DFA}}\left[n_{\uparrow}, 0\right]$ & 15,17 \\
\hline $\mathrm{CC} 0+$ prSCAN & $E^{\mathrm{CC} 0}+2 E_{c \uparrow \uparrow}^{\mathrm{rSCAN}}\left[n_{\uparrow}, n_{\downarrow}\right]$ & 14,20 \\
\hline $\mathrm{CC} 0+\operatorname{trSCAN}$ & $E^{\mathrm{CC} 0}+3 E_{c \uparrow \uparrow}^{\mathrm{rSCAN}}\left[n_{\uparrow}, n_{\downarrow}\right]$ & 17,20 \\
\hline $\mathrm{LC}-\mathrm{CC} 0+\mathrm{pDFT}$ & $E^{\mathrm{CC} 0}+2 E_{c}^{\mathrm{sr}-\mathrm{DFA}}\left[n_{\uparrow}, 0\right]+2 E_{c \uparrow \uparrow}^{\mathrm{Ir}-\mathrm{dRPA}}$ & $14,25-31$ \\
\hline $\mathrm{LC}-\mathrm{CC} 0+\mathrm{tDFT}$ & $E^{\mathrm{CC} 0}+3 E_{c}^{\mathrm{sr}-\mathrm{DFA}}\left[n_{\uparrow}, 0\right]+3 E_{c \uparrow \uparrow}^{\mathrm{lr}-\mathrm{dRPA}}$ & $17,15,25-31$ \\
\hline $\mathrm{LC}-\mathrm{CC} 0+$ prSCAN & $E^{\mathrm{CC} 0}+2 E_{c \uparrow \uparrow}^{\mathrm{sr}-\mathrm{rSCAN}}\left[n_{\uparrow}, n_{\downarrow}\right]+2 E_{c \uparrow \uparrow}^{\mathrm{lr}-\mathrm{dRPA}}$ & $14,20,25-31$ \\
\hline $\mathrm{LC}-\mathrm{CC} 0+\operatorname{trSCAN}$ & $E^{\mathrm{CC} 0}+3 E_{c \uparrow \uparrow}^{\mathrm{sr}-\mathrm{rSCAN}}\left[n_{\uparrow}, n_{\downarrow}\right]+3 E_{c \uparrow \uparrow}^{\mathrm{lr}-\mathrm{dRPA}}$ & $17,20,25-31$ \\
\hline
\end{tabular}

functionals 25. Note that we have used the decomposition by Stoll et al. 71] (Eq. 15) for determining the effect of considering only parallel spin correlation on the scaling factor; the Gori-Giorgi-Perdew 75] spin resolution can't be used for this because the $F_{\sigma \sigma^{\prime}}$ fractions (see Section 2.4) are independent of $\mu$. Nonetheless, $\epsilon_{c \uparrow \uparrow}^{\mathrm{MGGA}}$ may still be evaluated with the spin resolution of Eq. 20 for the SCAN functional. Also, both $\epsilon_{c, \mu}^{\mathrm{sr}-\mathrm{LDA}}\left(n_{\uparrow}, 0\right)$ and $\epsilon_{c}^{\mathrm{LDA}}\left(n_{\uparrow}, 0\right)$ are expected to exaggerate equal-spin correlation, resulting in error cancellation on the ratio that defines the scaling. Lastly, Eq. 31 maintains the $E_{c \uparrow \uparrow}^{\mathrm{sr}, \mu}=0$ condition for two-electron singlets as long as $\epsilon_{c \uparrow \uparrow}^{\mathrm{MGGA}}$ be evaluated with an adequate meta-GGA.

The separation of the electron-electron interaction with Eq. 21 also requires one to define a range-separation parameter $\mu$. In the case of standard LC-KS-DFT, where Eq. 21 is used to divide the exchange interaction only, the optimal $\mu$ is highly system and property dependent $96-$ 100. However, here we are interested in splitting the correlation in SR and LR, and in this case $\mu$ is more universal: Fromager et al. 101, 102 have demonstrated, based on physical arguments and numerical experiments, that the optimal $\mu$ for evaluating the SR correlation with semilocal DFAs is in the vicinity of $\mu \approx 0.4 \mathrm{au}$. Therefore, we set $\mu=0.4$ au in all of our calculations (note that this value is widely used in LC-KS-DFT functionals such as, e.g., LC- $\omega$ PBE [83]). Additionally, by fixing $\mu$, size consistency and extensivity are preserved; system-dependent definitions of $\mu$ not all can guarantee this [100, 103]. We note that it is also possible to define a physically-motivated local $\mu(r)$, but this has the caveat that locally-range separated hybrids are difficult and expensive to evaluate [104, 105].

Lastly, we remark that range separation using longrange dRPA correlation is one way to introduce the long-range part of the van der Waals interaction in CC0+DFT; other approaches are possible. For example, specialized van der Waals functionals of varying degree of empiricism have been developed by various groups (see, e.g., Refs. 106 109 and references therein). These can be used in $\mathrm{CC} 0+\mathrm{DFT}$ as long as meaningful spin resolution for the correlation of the functional exist.

\section{F. Summary of methods and notation}

The possible $\mathrm{CC} 0+\mathrm{DFT}$ combinations presented here and their corresponding closed-shell energy expressions are summarized in Table[1/(in this work, we deal only with closed shells). All these methods have been implemented in a development version of GAUSSIAN 110 . A variant called $\mathrm{CC} 0+E_{c}^{\mathrm{DFT}}$ is also introduced where the full $E_{c}^{\mathrm{DFA}}\left[n_{\uparrow}, n_{\downarrow}\right]$ is added to the CC0 energy. The purpose of including $\mathrm{CC} 0+E_{c}^{\mathrm{DFT}}$ is to assess the effects of double counting, and how well do improved $\mathrm{CC} 0+\mathrm{DFT}$ combinations eliminate this problem. The explanation of the notation for the rest of the methods is as follows: A "p" before the functional name indicates that only parallel spin correlation from the DFA - and dRPA, for the methods that include it - is added to $\mathrm{CC} 0$; the " $\mathrm{t}$ " that the full triplet-component of $T_{2}$ is added (see Section 2.2). The variant of SCAN called "rSCAN" uses the spin resolution from Eq.20, rather than that of Eq. 15. Finally, the "LC" prefix (which stands for "long-range corrected") specifies that the short- and long-range correlation terms are evaluated with the DFA and the dRPA, respectively. The long-range correction used here should not be confused with the one used in standard LC-KS-DFT; the former affects only the correlation, while the latter affects only the exchange. The exchange does not need corrections in $\mathrm{CC} 0+\mathrm{DFT}$ as it is calculated with the wavefunction method. We also remind the reader that the calculations are carried out in a non-self-consistent, post-CC0 manner; a self-consistent implementation is possible but not the focus of this exploratory paper. 


\section{RESULTS AND DISCUSSION}

\section{A. Description of short-range dynamic correlation}

For testing the $\mathrm{CC} 0+\mathrm{DFT}$ description of short-range dynamic correlation, we consider the equilibrium distances $\left(R_{e}\right)$ and harmonic vibrational frequencies $\left(\omega_{e}\right)$ of a set of ten first- and second-row diatomics that has been studied in previous MR+DFT works [14, 111]. These molecules are listed in Table II they comprise wellknown examples of single- and multiple-bonds, homonuclear and heteronuclear diatomics; accurate experimental data are available from established databases [112, 113. The motivation for studying $R_{e}$ and $\omega_{e}$ is as follows: $E_{c}^{\text {DFA }}$ increases in magnitude as the interatomic distance is reduced, increasing the bond strength. Because $\mathrm{CC} 0+E_{c}^{\mathrm{DFT}}$ is deliberately constructed to have double counting from $E_{c}^{\mathrm{DFA}}$, this approach should predict too short bond lengths and too high frequencies. If the rest of the $\mathrm{CC} 0+\mathrm{DFT}$ combinations in Table I really work, then these problems would disappear and improvement over CC0 should be observed.

The bond lengths predicted by BD0 and BD0+DFT are compared with accurate data in Table II results by CCD0 and CCD0+DFT are highly similar to those of their corresponding BD0 counterparts. Likewise, LC$\mathrm{CC} 0+\mathrm{DFT}$ and $\mathrm{CC} 0+\mathrm{DFT}$ are not significantly different in this case. Note that the $\mathrm{H}_{2}$ molecule, for which BD0 is exact, is included among the benchmark set. The accurate data are from experiments [112, 113] and the difference with BD0 for $\mathrm{H}_{2}(0.001 \AA)$ may be considered as an estimate of the effects of basis set incompleteness (and, in principle, also the Born-Oppenheimer approximation). As expected from the discussion above, BD0 $+E_{c}^{\mathrm{DFT}}$ consistently underestimates the bond lengths (mean error, $\mathrm{ME}=-0.015 \AA$ ), while the BD0+DFT methods that avoid double counting do not do this and furthermore improve upon BD0: the mean absolute errors (MAEs) of the different BD0+DFT combinations are 2-4 times smaller than the MAE of BD0 $(0.013 \AA)$, all providing very good results. Thus, the $\mathrm{CC} 0+\mathrm{DFT}$ methods appear to be working as intended in the prediction of equilibrium distances. Perhaps the only unexpected result is that there does not appear to be much difference between the different spin resolutions of SCAN (pSCAN and prSCAN), or between adding only parallel spin correlation or the full triplet component of $T_{2}$ (prSCAN and trSCAN). We also note that most of the largest errors occur in $\mathrm{Li}_{2}$. This diatomic is somewhat challenging to describe because, even at equilibrium, there may be some static correlation present 21]. Nevertheless, the errors for the $R_{e}$ of $\mathrm{Li}_{2}$ obtained here are much smaller than those reported for $\mathrm{MCSCF}$ (error $=0.258 \AA$ ) ) and MC$\mathrm{SCF}+\mathrm{DFT}$ (error $\approx 0.150 \stackrel{\circ}{A}$ ) in Ref. 14 .

General observations are mostly similar for the harmonic vibrational frequencies shown in Table III. Again, we exclude $\mathrm{LC}-\mathrm{CC} 0+\mathrm{DFT}$ data because the effect of the long-range correction with dRPA correlation is negligi- ble on the calculated frequencies. A difference though is that there is more variation between BD0+DFT and CCD0+DFT, and hence we include data for both in this Table. The effect of double counting is as expected and $\mathrm{CC} 0+E_{c}^{\mathrm{DFT}}$ consistently overestimates the frequencies $\left(\mathrm{ME}=\mathrm{MAE} \approx 70-80 \mathrm{~cm}^{-1}\right.$ with TPSS, $40-60 \mathrm{~cm}^{-1}$ with SCAN). Results from CCD0 and BD0 are close to each other $\left(\mathrm{MAE}=22 \mathrm{~cm}^{-1}\right.$ for both), but adding pTPSS to the former worsens results $\left(\mathrm{MAE}=42 \mathrm{~cm}^{-1}\right.$ ) while it does not for the latter $\left(\mathrm{MAE}=25 \mathrm{~cm}^{-1}\right)$. Adding pSCAN, prSCAN, or trSCAN to CCD0 gives similar or slightly worse results than CCD0; for BD0, predictions become similar or better. The densities from the approximate Brueckner orbitals may therefore be more adequate inputs for $E_{c}^{\mathrm{DFA}}$ than the RHF densities. This observation may be related to the assertions about the physical relevance of Brueckner orbitals - connections to DFT [114] and even the Kohn-Sham orbitals [115] have been suggested in the literature. Note also that the exact Brueckner determinant can be defined as the Slater determinant having the largest overlap with FCI, and thus the densities can be presumed to be of better quality than those from RHF. Overall, all of the combinations of CCD0 or BD0 with SCAN give satisfactory results for both bond lengths and frequencies; mixtures with TPSS are also reliable for these properties except for CCD0+pTPSS, which tends to overestimate the frequencies.

As noted above, it is rather surprising that different spin resolutions, or whether one uses pDFT or tDFT, have little effect on the calculated bond lengths and frequencies. However, these properties depend on relative energies only; total energies are certain to be affected by factors such as the incorporation of parallel spin vs. triplet pairing channel correlation (i.e., pDFT vs. tDFT). It is then logical to ask which of the different flavors of $\mathrm{CC} 0+\mathrm{DFT}$ provides more accurate total energies. Table IV compares the accurate [116] energy for the Neon atom with BD0 and BD0+DFT estimates; results from CCD0 and CCD0+DFT differ by less than 1 milihartree from their BD0 analogues. The correlation missed by BD0 in Ne is substantial (120 milihartrees). If raw $E_{c}^{\mathrm{DFT}}\left[n_{\uparrow}, n_{\downarrow}\right]$ is added, results are worsened due to overcorrelation (error $\approx-225$ milihartrees). In contrast, adding only parallel spin correlation improves the total energy, although underestimating it (errors $=41$ and 20 milihartrees for pTPSS and pSCAN, respectively). The best agreement with the accurate energy is obtained when the correlation from the full triplet pairing channel is added to BD0 and using the spin resolution of Eq. 20 (errors $=2$ and -10 milihartrees for tTPSS, and trSCAN, respectively), but there is overcorrelation if the spin resolution of Stoll et al. [71] is used with SCAN (error = -29 milihartrees for tSCAN). These observations are in line with the theoretical arguments from Sections 2.2 and 2.4 ; i.e., that BD0 misses the full triplet component of $T_{2}$, and that the resolution by Stoll et al. [71 exaggerates same-spin correlation in most regimes of the uniform 
TABLE II: Accurate [12, 113] equilibrium distances $\left(R_{e}\right.$, in $\left.\stackrel{\AA}{A}\right)$ for a set of diatomics and the deviations (calculated - accurate, in miliangstroms) from these distances for BD0 and BD0+DFT methods using a Cartesian 6-311++G(2df,2p) basis. ME is the mean error and MAE the mean absolute error. Results from CCD0 and CCD0+DFT are very similar.

\begin{tabular}{|c|c|c|c|c|c|c|c|c|}
\hline \multirow[b]{2}{*}{ Molecule } & \multirow[b]{2}{*}{ Accurate } & \multirow[b]{2}{*}{ BD0 } & \multicolumn{6}{|c|}{ BD0+ } \\
\hline & & & $E_{c}^{\mathrm{TPSS}}$ & $E_{c}^{\mathrm{SCAN}}$ & pTPSS & pSCAN & prSCAN & trSCAN \\
\hline $\mathrm{H}_{2}$ & 0.741 & 1 & -2 & 0 & 1 & 1 & 1 & 1 \\
\hline $\mathrm{LiH}$ & 1.596 & 6 & -10 & -12 & 3 & -2 & 1 & -5 \\
\hline $\mathrm{HF}$ & 0.917 & 1 & -10 & -5 & -5 & -1 & -1 & -2 \\
\hline $\mathrm{HCl}$ & 1.275 & 7 & -7 & -2 & 0 & 3 & 4 & 2 \\
\hline $\mathrm{Li}_{2}$ & 2.673 & -28 & -28 & -50 & -2 & -20 & -11 & -28 \\
\hline $\mathrm{C}_{2}$ & 1.243 & 4 & -9 & -6 & -1 & 0 & 0 & -2 \\
\hline $\mathrm{N}_{2}$ & 1.098 & 1 & -8 & -5 & -2 & -1 & -1 & -2 \\
\hline $\mathrm{F}_{2}$ & 1.412 & 15 & -34 & -26 & -14 & -6 & -2 & -11 \\
\hline $\mathrm{Cl}_{2}$ & 1.988 & 41 & -19 & -9 & 2 & 12 & 20 & 9 \\
\hline $\mathrm{ClF}$ & 1.628 & 25 & -19 & -8 & 0 & 8 & 12 & 5 \\
\hline $\mathrm{ME}$ & & 7 & -15 & -12 & -2 & -1 & 2 & -3 \\
\hline MAE & & 13 & 15 & 12 & 3 & 5 & 5 & 7 \\
\hline
\end{tabular}

TABLE III: Accurate [112, 113] harmonic vibrational frequencies $\left(\omega_{e}\right.$, in $\left.\mathrm{cm}^{-1}\right)$ for a set of diatomics and the deviations (calculated - exact) from these values for CC0 and CC0+DFT methods using a Cartesian 6-311++G(2df,2p) basis. ME is the mean error and MAE the mean absolute error.

\begin{tabular}{|c|c|c|c|c|c|c|c|c|}
\hline \multirow[b]{2}{*}{ Molecule } & \multirow[b]{2}{*}{ Accurate } & \multirow[b]{2}{*}{ CCD0/BD0 } & \multicolumn{6}{|c|}{$\mathrm{CCD} 0+/ \mathrm{BD} 0+$} \\
\hline & & & $E_{c}^{\mathrm{TPSS}}$ & $E_{c}^{\mathrm{SCAN}}$ & pTPSS & pSCAN & prSCAN & trSCAN \\
\hline $\mathrm{H}_{2}$ & 4401 & $23 / 11$ & $65 / 60$ & $42 / 23$ & $23 / 11$ & $23 / 11$ & $23 / 11$ & $23 / 11$ \\
\hline $\mathrm{LiH}$ & 1406 & $-6 /-3$ & $32 / 30$ & $39 / 31$ & $0 / 4$ & $0 / 3$ & $-3 /-11$ & $9 / 0$ \\
\hline $\mathrm{HF}$ & 4138 & $36 / 19$ & $160 / 156$ & $103 / 84$ & $102 / 84$ & $22 / 24$ & $44 / 24$ & $58 / 38$ \\
\hline $\mathrm{HCl}$ & 2991 & $-38 /-38$ & $70 / 65$ & $35 / 21$ & $7 / 21$ & $-22 /-21$ & $-32 /-47$ & $-17 /-29$ \\
\hline $\mathrm{Li}_{2}$ & 351 & $-3 / 32$ & $19 / 18$ & $11 / 10$ & $9 / 7$ & $9 / 26$ & $2 / 16$ & $12 / 5$ \\
\hline $\mathrm{C}_{2}$ & 1855 & $22 / 12$ & $106 / 87$ & $77 / 58$ & $89 / 51$ & $40 / 29$ & $40 / 26$ & $50 / 36$ \\
\hline $\mathrm{N}_{2}$ & 2359 & $36 / 19$ & $108 / 87$ & $77 / 54$ & $63 / 40$ & $37 / 25$ & $44 / 21$ & $51 / 29$ \\
\hline $\mathrm{F}_{2}$ & 917 & $17 /-31$ & $132 / 87$ & $110 / 65$ & $81 / 8$ & $64 / 9$ & $52 / 0$ & $76 / 22$ \\
\hline $\mathrm{Cl}_{2}$ & 560 & $-19 /-19$ & $45 / 41$ & $32 / 27$ & $22 / 17$ & $7 / 10$ & $-2 /-6$ & $10 / 5$ \\
\hline $\mathrm{ClF}$ & 786 & $-24 /-34$ & $54 / 41$ & $36 / 18$ & $20 / 6$ & $7 / 0$ & $-4 /-15$ & $12 /-4$ \\
\hline ME & & $4 /-3$ & $79 / 67$ & $56 / 39$ & $42 / 25$ & $19 / 11$ & $16 / 2$ & $28 / 11$ \\
\hline MAE & & $22 / 22$ & $79 / 67$ & $56 / 39$ & $42 / 25$ & $23 / 16$ & $25 / 18$ & $32 / 18$ \\
\hline
\end{tabular}

electron gas.

Another test for dynamic correlation based on relative energies, but more sensitive than the bond lengths and frequencies above, is the description of proton affinities. When a proton is added to a neutral molecule, the density is redistributed and dynamic correlation changes subtly. This effect is reasonably well described by density functionals, which have average errors of about 1-3 $\mathrm{kcal} / \mathrm{mol}$ in small molecules [117, 118. Table V] compares the experimental (ZPE-corrected) proton affinities of eight molecules with those calculated by TPSS, SCAN, BD0, and BD0+DFT. The geometries (which are optimized at the MP2/6-31G(2df,p) level) and reference data were taken from Ref. 118; the effects of geometric relaxation are known to be negligible [117. Again, we focus on BD0+DFT methods because CCD0+DFT results are very similar and the long-range correction of LC-BD0+DFT has little effect on the proton affinities. Pure BD0 tends to overestimate the proton affinities (ME
$=2.58 \mathrm{kcal} / \mathrm{mol}$ ) and has the largest MAE of all methods $(2.69 \mathrm{kcal} / \mathrm{mol})$. TPSS results are similar $(\mathrm{ME}=\mathrm{MAE}=$ $2.65 \mathrm{kcal} / \mathrm{mol}$ ), but SCAN is considerably better (MAE $=1.01 \mathrm{kcal} / \mathrm{mol})$. Previous works noted that TPSS did not improve upon its Perdew-Kurth-Zupan-Blaha [119] predecessor in the prediction of proton affinities [117. The fact that SCAN is better than TPSS at describing the effect of protonization is reflected in BD0+DFT: the $\mathrm{BD} 0+\mathrm{TPSS}$ errors $(\mathrm{MAE} \approx 2.3 \mathrm{kcal} / \mathrm{mol}$ ) are $2-3$ times larger than the $\mathrm{BD} 0+\mathrm{SCAN}$ errors. The best results are obtained when the full triplet-pairing component of $T_{2}$ is added to BD0 using the spin resolution of Section $2.4(\mathrm{BD} 0+\operatorname{trSCAN}, \mathrm{MAE}=0.66 \mathrm{kcal} / \mathrm{mol})$. The observations here point toward a relationship between the accuracies of the DFA and BD0+DFA for a given property. Note, however, that not any functional can be utilized in $\mathrm{CC} 0+\mathrm{DFT}$. For example, the Lee-YangParr [120] (LYP) functional models all correlation as being opposite-spin [121], so that there is no parallel-spin 
TABLE IV: Accurate [16] total energy for the Neon atom (Hartrees) and deviations (calculated - exact, in milihartrees) from this value for BD0 and BD0+DFT methods using a Cartesian cc-pwCV5Z basis. Results from CCD0 and CCD0+DFT differ by less than 1 milihartree from their BD0 counterparts.

\begin{tabular}{lccccccccr}
\hline & & \multicolumn{7}{c}{ BD0+ } \\
\cline { 2 - 9 } Accurate & BD0 & $E_{c}^{\text {TPSS }}$ & $E_{c}^{\text {SCAN }}$ & pTPSS & tTPSS & pSCAN & tSCAN & prSCAN & trSCAN \\
\hline-128.938 & 120 & -229 & -224 & 41 & 2 & 20 & -29 & 33 & -10 \\
\hline
\end{tabular}

TABLE V: Accurate proton affinities $\left(\mathrm{PA}=E(M)-E\left(M H^{+}\right)\right.$, in kcal $\left./ \mathrm{mol}\right)$ for eight molecules and deviations (calculated exact, in $\mathrm{kcal} / \mathrm{mol}$ ) from these values for TPSS, SCAN, BD0, and BD0+DFT using a Cartesian MG3S basis. The TPSS and accurate data are from Ref. [118. Results from CCD0 and CCD0+DFT are very similar to those from their BD0 counterparts.

\begin{tabular}{|c|c|c|c|c|c|c|c|c|c|c|}
\hline \multirow[b]{2}{*}{ Molecule } & \multirow[b]{2}{*}{ Accurate } & \multirow[b]{2}{*}{ TPSS } & \multirow[b]{2}{*}{ SCAN } & \multirow[b]{2}{*}{ BD0 } & \multicolumn{6}{|c|}{ BD0+ } \\
\hline & & & & & pTPSS & tTPSS & pSCAN & tSCAN & prSCAN & trSCAN \\
\hline $\mathrm{NH}_{3}$ & 211.90 & 1.70 & 0.72 & 2.84 & 3.53 & 3.88 & 1.22 & 0.41 & 1.70 & 1.13 \\
\hline $\mathrm{H}_{2} \mathrm{O}$ & 171.80 & 0.40 & 0.24 & 1.44 & 2.43 & 2.93 & 0.39 & -0.14 & 0.69 & 0.31 \\
\hline $\mathrm{C}_{2} \mathrm{H}_{2}$ & 156.60 & 4.60 & 3.83 & 2.89 & 4.01 & 4.56 & 0.95 & -0.02 & 1.30 & 0.50 \\
\hline $\mathrm{SiH}_{4}$ & 156.50 & 3.10 & -0.34 & 2.75 & 0.46 & -0.68 & 0.24 & -1.01 & 0.84 & -0.12 \\
\hline $\mathrm{PH}_{3}$ & 193.10 & 2.60 & -0.02 & 4.55 & 1.04 & -0.71 & -0.26 & -2.66 & 1.09 & -0.65 \\
\hline $\mathrm{H}_{2} \mathrm{~S}$ & 173.70 & 3.30 & 1.45 & 3.72 & 2.74 & 2.24 & 1.04 & -0.30 & 1.79 & 0.83 \\
\hline $\mathrm{HCl}$ & 137.10 & 3.10 & 1.50 & 2.92 & 3.18 & 3.31 & 1.42 & 0.68 & 1.83 & 1.29 \\
\hline $\mathrm{H}_{2}$ & 105.90 & 2.40 & -0.01 & -0.44 & -0.44 & -0.44 & -0.44 & -0.44 & -0.44 & -0.44 \\
\hline $\mathrm{ME}$ & & 2.65 & 0.92 & 2.58 & 2.12 & 1.89 & 0.57 & -0.43 & 1.11 & 0.36 \\
\hline MAE & & 2.65 & 1.01 & 2.69 & 2.23 & 2.34 & 0.75 & 0.71 & 1.22 & 0.66 \\
\hline
\end{tabular}

correlation that can be extracted from LYP.

\section{B. Description of long-range dynamic correlation}

In all the benchmarks that we have hitherto discussed, the use of $\mathrm{LC}-\mathrm{CC} 0+\mathrm{DFT}$ over $\mathrm{CC} 0+\mathrm{DFT}$ has negligible impact on the results. However, the effect of the longrange, dRPA-based, correction can be dramatic on the description of van der Waals interactions. One such example, the dissociation of a Helium dimer, is shown in Figure 1. The accurate data shown there has been taken from Ref. [122]. Standard Brueckner doubles (BD) correctly describes the dissociation profile of $\mathrm{He}_{2}$. The correlation present in BD but not in BD0 is crucial in this case; the latter is repulsive in the region near the correct minimum. The reason for the failure of $\mathrm{BD} 0$ for long-range interactions can be explained as follows: BD0 misses equalspin correlation (plus an identical-in-magnitude $m=0$ component of $\left.T_{2}^{[1]}\right)$ which, in the short-range, is generally much smaller than the opposite-spin contributions because same-spin electrons avoid each other due to the Pauli principle (antisymmetry of the wavefunction). In the long-range, however, electrons are far apart from each other and their spin is no longer relevant on their dynamic correlation. Hence, the correlation missing in BD0 becomes more important in the long range.

From Figure1 we also see that BD0+TPSS adds a negligible contribution to the BD0 description of the $\mathrm{He}_{2}$ dissociation; the same-spin TPSS correlation is practically zero along the whole curve. Surprisingly, BD+SCAN

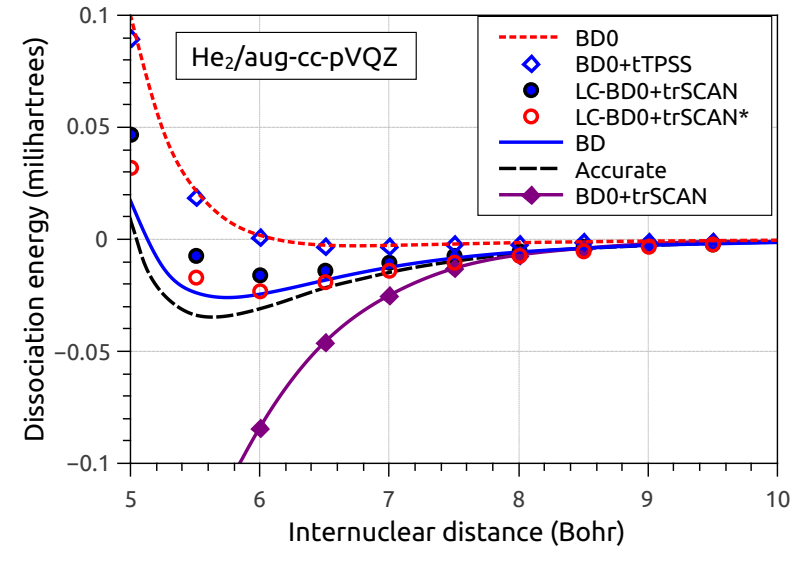

FIG. 1: Counterpoise-corrected dissociation energy profiles for the Helium dimer calculated by various methods using a Cartesian aug-cc-pVQZ basis. LC-BD0+trSCAN uses $\mu=0.4$ au.; $\mathrm{BD} 0+\mathrm{tSCAN}$ has the same behavior as BD0+trSCAN. The curve marked as LC-BD0+trSCAN* uses the semiempirical prefactor of 1.5 on the dRPA correlation of Ref. [85]. The accurate data are from Ref. [122].

is radically different from BD0+TPSS, but fails badly in an opposite way: SCAN exaggerates the long-range parallel-spin correlation and leads to far too much binding. This wrong behavior is corrected when the DFA correlation is evaluated in the short-range only and complemented with dRPA correlation in the long range. The LC-BD0+trSCAN and BD curves are close to each other, 
TABLE VI: Accurate binding energy for the Neon dimer compared with counterpoise-corrected calculations by BD0 and BD0+DFT methods at the experimental equilibrium distance of 5.841 au using a Cartesian aug-cc-pVQZ basis. The geometries and accurate binding energy were taken from Ref. [123]. All energies are in $\mathrm{kcal} / \mathrm{mol}$.

\begin{tabular}{lc}
\hline Method & Binding Energy \\
\hline Accurate & 0.08 \\
BD0 & -0.03 \\
BD0+pTPSS & -0.03 \\
BD0+tTPSS & -0.02 \\
BD0+pSCAN & 0.03 \\
BD0+tSCAN & 0.06 \\
BD0+prSCAN & 0.01 \\
BD0+trSCAN & 0.03 \\
LC-BD0+pTPSS & 0.00 \\
LC-BD0+tTPSS & 0.02 \\
LC-BD0+prSCAN & 0.01 \\
LC-BD0+trSCAN & 0.03 \\
LC-BD0+tTPSS* & 0.05 \\
LC-BD0+trSCAN* & 0.05 \\
${ }^{*}$ Using the semiempirical prefactor of 1.5 on the \\
dRPA correlation of determined on Ref. [85].
\end{tabular}

indicating that adding the full triplet component of $T_{2}$ via our SR-SCAN/LR-dRPA model correctly incorporates the correlation that is missing in BD0. It is worth pointing out that Janesko et al. [85] found that using an empirical scaling of 1.5 on the dRPA correlation improves the agreement of LC-LDA+dRPA with accurate data for noble gas dimers, and that here too we observe improvement if this factor is used (LC-BD0+trSCAN* curve). Note also that we do not show data for all possible method combinations in Figure 1 , but the performance of the techniques not shown can be inferred by the reader due to the fact that many BD0+DFT variants differ only by a multiplicative factor on the correlation added to BD0. For example, the LC-BD0+prSCAN curve (not shown in Figure 1) would be qualitatively similar to the LC-BD0+trSCAN one, but less accurate in quantitative terms.

On Table VI the binding energies of the Neon dimer calculated by $\mathrm{BD}$ and $\mathrm{BD} 0+\mathrm{DFT}$ at the experimental equilibrium distance of 5.841 au are compared with accurate estimates from standard databases [123]. Once more, BD0 fails to bind the noble gas dimer, and so do BD0+pTPSS and BD0+tTPSS. Remarkably though, all combinations of BD0 with SCAN bind $\mathrm{Ne}_{2}$, showing best agreement with the reference energy when the full triplet-pairing component of $T_{2}$ is included. When the long-range DFA correlation is replaced by dRPA correlation, variants using TPSS and SCAN all bind the dimer, but tSCAN-based methods are most accurate. LC-BD0+pTPSS provides the smallest binding energy $(0.004 \mathrm{kcal} / \mathrm{mol})$ and LC-BD0+trSCAN the largest one $(0.03 \mathrm{kcal} / \mathrm{mol})$. As occurs for $\mathrm{He}_{2}$, if the semiempiri-
TABLE VII: Accurate binding energies for ethylene $\left(\mathrm{C}_{2} \mathrm{H}_{4}\right)$ and acetylene $\left(\mathrm{C}_{2} \mathrm{H}_{2}\right)$ dimers compared with counterpoisecorrected calculations by BD0 and BD0+DFT methods using a Cartesian MG3S basis. The geometries and accurate data were taken from Ref. 123. All energies are in kcal $/ \mathrm{mol}$.

\begin{tabular}{lcc}
\hline & \multicolumn{2}{c}{ Binding Energy } \\
\cline { 2 - 3 } Method & $\left(\mathrm{C}_{2} \mathrm{H}_{2}\right)_{2}$ & $\left(\mathrm{C}_{2} \mathrm{H}_{4}\right)_{2}$ \\
\hline Accurate & 1.34 & 1.42 \\
BD0 & 0.38 & -0.42 \\
BD0+pTPSS & 0.64 & 0.07 \\
BD0+tTPSS & 0.76 & 0.31 \\
BD0+pSCAN & 1.13 & 1.03 \\
BD0+tSCAN & 1.50 & 1.76 \\
BD0+prSCAN & 0.95 & 0.23 \\
BD0+trSCAN & 1.24 & 0.56 \\
\hline
\end{tabular}

cal factor of 1.5 determined on Ref. 85 is used to scale $E_{c}^{\mathrm{dRPA}}$ the agreement between LC-BD0+tDFT and experiment is improved.

It should also be mentioned that there are some types of weak interactions that are captured by semilocal functionals, as these can describe the intermediate-range part of the van der Waals interaction [74, 124, 125. As an example, we consider the $\pi$ - $\pi$ interactions in ethylene and acetylene dimers with geometries from a standard dataset [123]. Accurate data for the binding energies of these species are compared with $\mathrm{BD} 0$ and $\mathrm{BD} 0+\mathrm{DFT}$ predictions on Table VII. At the accurate equilibrium distance, BD0 significantly underestimates the binding energies for both dimers and predicts only the acetylene dimer to be bound. All of the BD0 combinations with DFT improve the former's results and bind both dimers, but the best agreement with the reference data is given by $\mathrm{BD} 0+\mathrm{tSCAN}$ and $\mathrm{BD} 0+\operatorname{trSCAN}$. It was noted on Ref. 74] that SCAN was more accurate than TPSS for describing weak interactions; a consequence, most likely, of the appropriate norming of SCAN. Hence, we observe again a correlation between the adequacy of the DFA for a given property, and that of BD0+DFA methods for the same type of calculation.

In this Section, we have focused on $\mathrm{BD} 0+\mathrm{DFT}$ results; CCD0+DFT results have been omitted. The reason for this is that, in our experience, BD0+DFT and CCD0+DFT are largely similar when describing problems dominated by dynamic correlation (with BD0 being slightly better). Thus, the observations for CCD0-based methods are analogous to those of their BD0 counterparts and discussion about the former has been left out to avoid repetitiveness and long-windedness. Nevertheless, as we discuss next, there can be important differences between CCD0 and BD0 when static correlation is involved. 

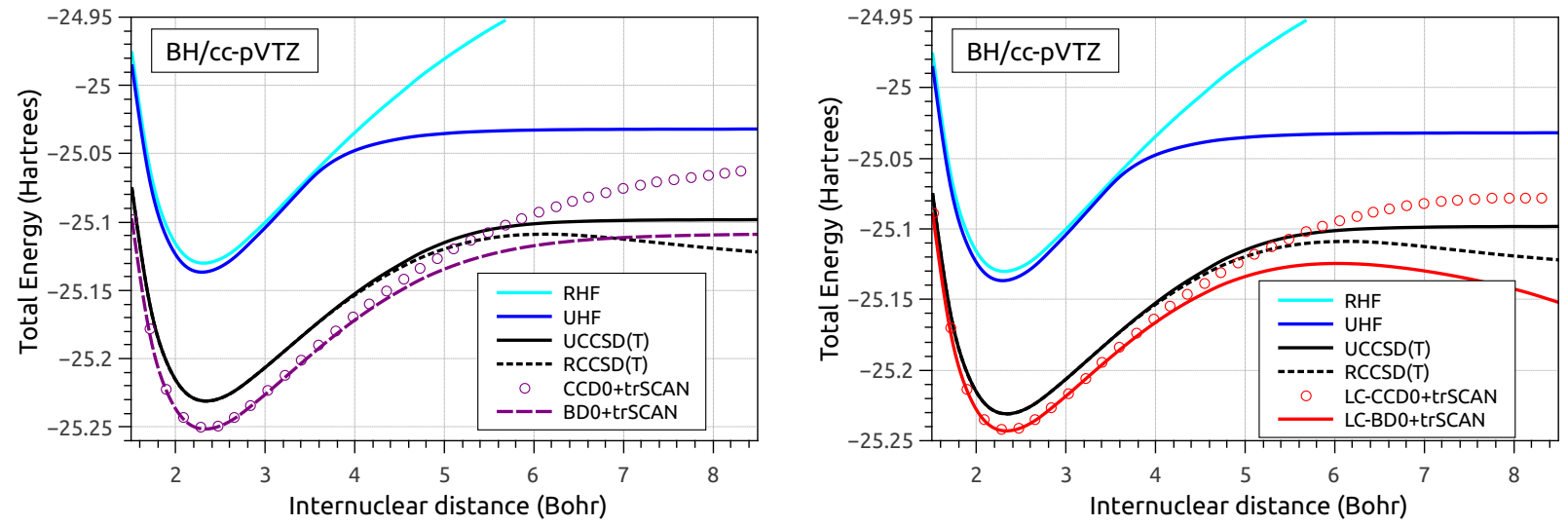

FIG. 2: Dissociation energy profiles for boron hydride calculated by various methods using a Cartesian cc-pVTZ basis.

\section{Simultaneous description of static and dynamic correlation}

Molecular dissociations are one of the paradigms of static correlation in quantum chemistry. Figure 2 shows the dissociation energy curves for boron hydride calculated by various standard and $\mathrm{CC} 0+\mathrm{rSCAN}$ methods; $\mathrm{CC} 0+\mathrm{TPSS}$ and $\mathrm{CC} 0+\mathrm{SCAN}$ variants are similar but slightly higher and lower, respectively, in energy than $\mathrm{CC} 0+\mathrm{rSCAN}$. The UCCSD $(\mathrm{T})$ (unrestricted CCSD with perturbative triples) data in this Figure may be considered as an accurate reference, as this technique is considerably parallel to FCI for this system 126] (nonparallelity error $\approx 2 \mathrm{kcal} / \mathrm{mol}$ ). Note, however, that restricted $\mathrm{CCSD}(\mathrm{T})$ experiences a breakdown at $\mathrm{B}-\mathrm{H}$ distances greater than about $5.5 \mathrm{au}$. As noted in the previous sections, CCD0- and BD0-based methods are highly similar when the problem is dominated by dynamic correlation (in this case, near equilibrium, although the existence of a UHF solution slightly lower in energy than RHF suggests the presence of some static correlation too; similar observations have been noted by Pollet et al. [21]). However, this is no longer true at large bond lengths, where CCD0 becomes significantly higher in energy than BD0. In general, the BD0 description of bond breaking is significantly better than that of CCD0. This is easily understood by considering the dissociation of $\mathrm{H}_{2}$ : $\mathrm{BD} 0$ is exact but CCD0 is not because, in order to be exact for two-electron systems, singles contributions must be included either explicitly or via the Brueckner orbitals. As the total correlation increases when the bond is extended beyond the Coulson-Fischer point, the singles contributions that are missing in CCD0 become more important. This analysis extends to other chemical bonds in general.

Although the BD0+trSCAN curve in Fig. 2 provides a very good description of the $\mathrm{BH}$ dissociation (and without resorting to symmetry breaking, like $\operatorname{UCCSD}(\mathrm{T})$ ), LC-BD0+trSCAN exhibits wrong behavior at large bond lengths. The dRPA correlation increases too much beyond $R_{\mathrm{B}-\mathrm{H}} \approx 6 \mathrm{au}$, resulting in a "bump", similar to that of $\operatorname{RCCSD}(\mathrm{T})$, characteristic of common perturbative and RPA-based approaches. In the case of LCCCD0+trSCAN, the substantial dRPA correlation actually helps to correct, to a certain extent, the toohigh dissociation limit of CCD0+trSCAN. When breaking multiple bonds, coupled cluster methods for strong correlation - CCD0, BD0, and pCCD - all tend to a toohigh energy limit (sometimes higher than UHF, e.g., in $\mathrm{N}_{2}$; see Figure 3). Thus, adding dRPA correlation in a manner similar to the one done here may provide a route to alleviate this problem of singlet-paired coupled cluster techniques. This, however, requires further investigation that is beyond the scope of the present paper. In the case of the dissociation of $\mathrm{N}_{2}$ shown in Figure 3, there also appears to be an improvement of the too-high dissociation energy limit of CCD0 by the long-range dRPA correlation. LC-CCD0+trSCAN is quite close energetically to UCCSD and binding energies are no longer exaggerated. The BD0+DFT curves are above LC-CCD0+DFT at large internuclear distances, but LC-BD0+DFT (not shown) leads to too much correlation at dissociation and a pronounced bump similar to that seen in boron hydride. Note also that although this is one of the cases in which BD0 goes to a too high limit, CCSD and CCSDT fail very badly while BD0+DFT provides a reasonable description of the dissociation (and is also accurate near equilibrium, as evidenced by the data in Tables II] and III).

Another problem for which traditional coupled cluster fails badly is the deformation of $\mathrm{H}_{4}$ from a rectangular to a square geometry [2]. Figure 4 shows this model (which has been studied extensively [127-131]) schematically. The $\mathrm{H}$ atoms are confined to a circle of radius 3.284 au and preserve $D_{2 h}$ symmetry, so that the geometry depends on a single parameter: the angle $\Theta$ that bisects two lines connecting opposing $\mathrm{H}$ atoms. This Figure also shows the dependence of the energy on $\Theta$ for various methods, including FCI data from Ref. [131. It is seen that CCSD predicts a trend that is opposite to that of FCI, with a minimum at $\Theta=90^{\circ}$ (square geometry), 

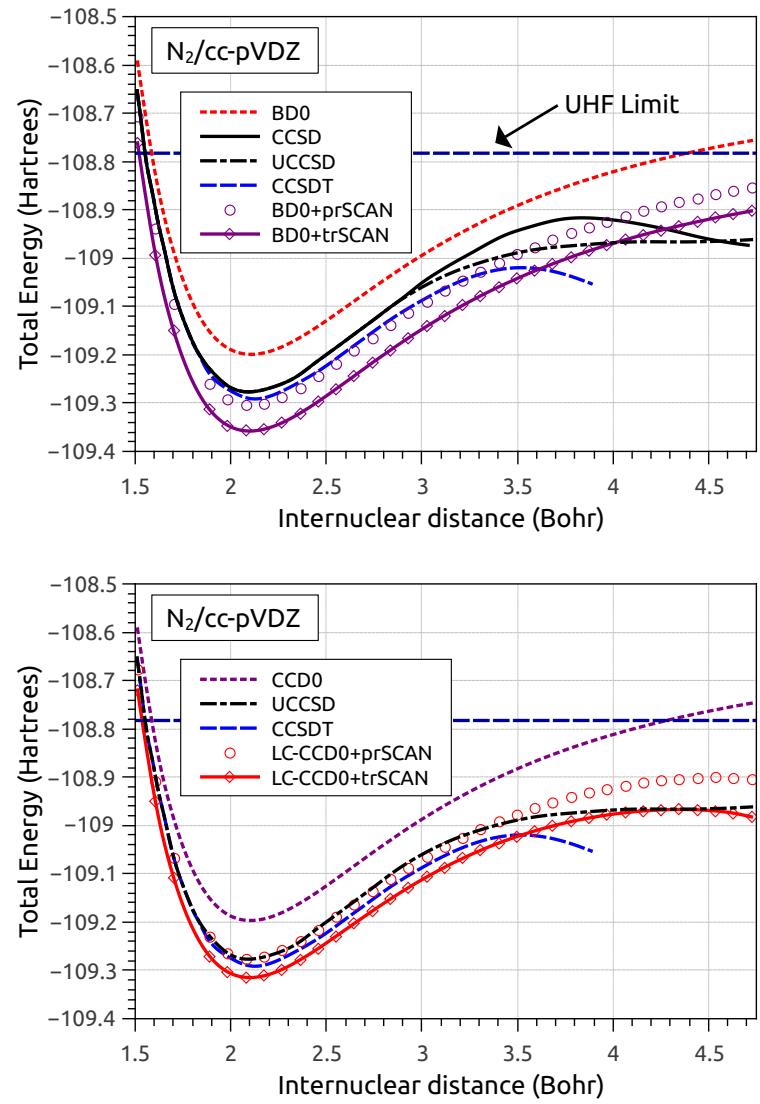

FIG. 3: Dissociation energy profiles for the $\mathrm{N}_{2}$ molecule calculated by various methods using a Cartesian cc-pVDZ basis.

where it overcorrelates the most. In contrast, BD0 and BD0+DFT methods have good qualitative and quantitative agreement with FCI, albeit they exhibit a discontinuity in the first derivative of the energy with respect to $\Theta$ at $\Theta=90^{\circ}$ that is not present in the FCI curve. Of the BD0+DFT methods, only BD0+rSCAN data are shown in Figure 4 but using TPSS gives very similar results, while BD0+SCAN yields slightly lower total energies.

A benchmark for which KS-DFT methods fail catastrophically is the Beryllium isoelectronic series (i.e., $X^{(Z-4)+}$ ions). In this system, the angular $s^{2} \rightarrow p^{2}$ static correlation increases linearly with increasing nuclear charge $Z$; this effect is poorly described by common DFAs (even if one tries to break spin symmetry, because the $X^{(Z-4)+}$ ions are RHF $\rightarrow$ UHF stable). Table VIII compares the accurate energies 132 for this series with energies from TPSS, SCAN, BD0, and their combinations; $\mathrm{CCD} 0+\mathrm{DFT}$ and $\mathrm{BD} 0+\mathrm{DFT}$ results are not significantly different in this case. The nonparallelity errors (NPE) - the difference between the maximum and minimum errors - are also provided as an estimate of how well is the linear trend of the correlation described. As expected, TPSS and SCAN fail badly to capture this trend with NPEs of 40.6 and 87.9 milihartrees, respectively. Much better results are given by BD0 $(\mathrm{NPE}=$
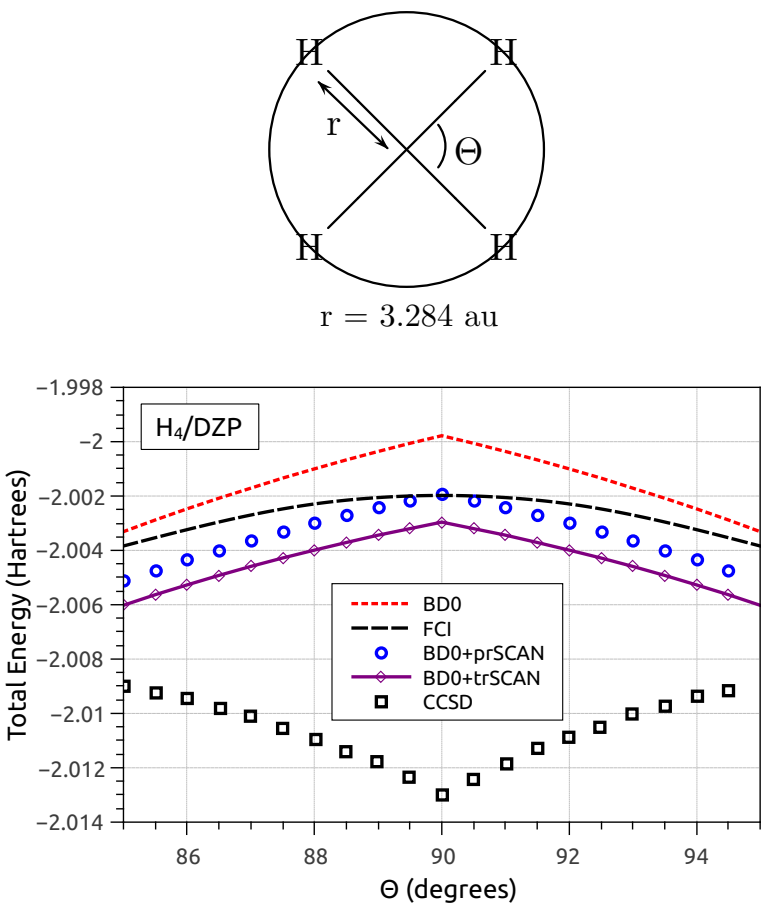

FIG. 4: Geometry of $\mathrm{H}_{4}$ on a circle with a radius of $3.284 \mathrm{au}$. Dependence of the total energy on the angle $\Theta$ for various methods using a Dunning DZP basis. The FCI data are from Ref. [131].

5.2 milihartrees), although there is underestimation of the energy due to the missing same-spin correlation (ME $=\mathrm{MAE}=7.1$ milihartrees $)$. All of the BD0+DFT combinations capture correctly the dependence of the energy with respect to $Z$, and are similar or better than BD0 with NPEs in the range of 2.0-7.3 milihartrees. The best results are given by BD0+pTPSS (ME, MAE, and NPE of $-2.5,2.5$, and 1.2 milihartrees, respectively). The BD0+DFT methods tend to overestimate the correlation, which may be a result of the approximate functional and/or of the resolution of the equal-spin correlation. Indeed, the latter appears to be a factor because $\mathrm{BD} 0+\mathrm{SCAN}$ overcorrelates more than $\mathrm{BD} 0+\mathrm{rSCAN}$. It is also possible that the overcorrelation in +tDFT methods for the $X^{(Z-4)+}$ ions be in part an artifact of the small size of the system; total energies for the isovalent $\mathrm{Mg}$ atom (Table IX) are most accurate with BD0+tTPSS and $\mathrm{BD} 0+\operatorname{trSCAN}$ and trends are similar to those observed for the Ne atom on Table IV] with BD0+DFT providing great improvement over BD0.

In the tests for static correlation thus far analyzed, BD0 is able to provide a qualitatively correct description of the problem at hand. A challenging benchmark for which this is not the case is the dissociation of the Beryllium dimer. This dissociation is chemically different from the breaking of typical bonds discussed above: $\mathrm{Be}_{2}$ has a formal (MO-theory) bond order of zero; it is weakly bound $(\approx 2 \mathrm{kcal} / \mathrm{mol}$ ) but not by dispersive van der Waals forces, but by a mixture of dynamic and static cor- 
TABLE VIII: Accurate 132 total energies (in Hartrees) for the Beryllium isoelectronic series and deviations (calculated exact, in milihartrees) from these values for TPSS, SCAN, BD0, and BD0+DFT using a Cartesian cc-pCVQZ basis. Results from CCD0 and CCD0+DFT are very similar to those from their BD0 counterparts.

\begin{tabular}{|c|c|c|c|c|c|c|c|c|c|c|}
\hline \multirow[b]{2}{*}{ Species } & \multirow[b]{2}{*}{ Accurate } & \multirow[b]{2}{*}{ TPSS } & \multirow[b]{2}{*}{ SCAN } & \multirow[b]{2}{*}{ BD0 } & \multicolumn{6}{|c|}{$\mathrm{BD} 0+$} \\
\hline & & & & & pTPSS & tTPSS & pSCAN & tSCAN & prSCAN & $\operatorname{trSCAN}$ \\
\hline$\overline{\mathrm{Be}}$ & -14.6674 & -4.2 & 17.3 & 4.0 & -2.7 & -6.0 & 6.0 & -11.1 & -3.7 & -7.5 \\
\hline $\mathrm{B}^{+}$ & -24.3498 & 3.0 & 31.8 & 6.0 & -2.4 & -6.5 & -6.8 & -13.2 & -4.2 & -9.3 \\
\hline $\mathrm{C}^{2+}$ & -36.5352 & 8.8 & 45.2 & 6.2 & -3.2 & -7.9 & -8.4 & -15.7 & -5.8 & -11.7 \\
\hline $\mathrm{N}^{3+}$ & -51.2234 & 15.6 & 59.9 & 7.1 & -3.0 & -8.0 & -8.8 & -16.7 & -6.2 & -12.9 \\
\hline $\mathrm{O}^{4+}$ & -68.4128 & 22.8 & 75.4 & 8.3 & -2.3 & -7.5 & -8.5 & -17.0 & -6.1 & -13.3 \\
\hline $\mathrm{F}^{5+}$ & -88.1022 & 29.5 & 90.2 & 8.7 & -2.2 & -7.7 & -8.9 & -17.7 & -6.6 & -14.2 \\
\hline $\mathrm{Ne}^{6+}$ & -110.2921 & 36.4 & 105.1 & 9.2 & -2.0 & -7.6 & -9.0 & -18.1 & -6.8 & -14.8 \\
\hline $\mathrm{ME}$ & & 16.0 & 60.7 & 7.1 & -2.5 & -7.3 & -8.1 & -15.6 & -5.6 & -12.0 \\
\hline MAE & & 17.2 & 60.7 & 7.1 & 2.5 & 7.3 & 8.1 & 15.6 & 5.6 & 12.0 \\
\hline NPE & & 40.6 & 87.9 & 5.2 & 1.2 & 2.0 & 3.0 & 7.1 & 3.1 & 7.3 \\
\hline
\end{tabular}

TABLE IX: Accurate [116] total energy for the Magnesium atom (Hartrees) and deviations (calculated - exact, in milihartrees) from this value for BD0 and BD0+DFT methods using a Cartesian cc-pwCV5Z basis.

\begin{tabular}{lccccccc}
\hline & & & & & & \\
Accurate & BD0 & pTPSS & tTPSS & pSCAN & tSCAN & prSCAN & trSCAN \\
\hline-200.053 & 143 & 48 & 1 & 24 & -36 & 38 & -15 \\
\hline
\end{tabular}

relation [133. Because of this, common coupled cluster and perturbation methods fail for this system; multireference wavefunctions may or may not work depending on the size of the active space and on how dynamic correlation is treated 133-135. Figure 5 shows that BD0 also fails badly for $\mathrm{Be}_{2}$; the potential energy curve is repulsive near the accurate equilibrium distance (determined by an explicitly correlated MR wavefunction, which agrees with experiment [134, 135]). In contrast, BD0+DFT methods bind the Be dimer, with BD0+tTPSS giving particularly good qualitative and quantitative agreement with the accurate reference data. In this case, BD0- and CCD0based provide similar results. LC-BD0+tTPSS affords a good description of the dissociation when the semiempirical prefactor of 1.5 on the dRPA correlation of Ref. 85] is used; otherwise the relative energy at the equilibrium distance may be too high (albeit there is still substantial improvement over BD0). It has been noted that connected triples are needed to obtain sizable bonding [136]; the ability of BD0+DFT to do this is thus a good indicative that the way in which the methods are mixed is adequate. The dissociation of $\mathrm{Be}_{2}$ is an example of the importance of the correlation missing in CC0 for obtaining qualitatively correct results in difficult cases where a simultaneous, balanced description of both static and dynamic correlation is necessary.

\section{CONCLUSIONS, OUTLOOK, AND POSSIBLE IMPROVEMENTS}

We have presented techniques to incorporate the correlation that is missing in singlet-paired coupled cluster via density functionals or combinations of these with the dRPA. These methods are nonemprical and physically motivated, and the benchmarks studied here demonstrate that they are capable of describing static and dynamic correlation (including long-range weak interactions) without symmetry breaking. Typical problems of $\mathrm{MR}+\mathrm{DFT}$ methods such as double counting and the symmetry dilemma are avoided in $\mathrm{CC} 0+\mathrm{DFT}$. In general, CCD0- and BD0-based methods provide similar results for weakly correlated systems, but $\mathrm{BD} 0+\mathrm{DFT}$ is preferable for problems with static correlation such as bond breaking. Similarly, both CC0+TPSS and CC0+SCAN yield good overall results, although SCAN is better at capturing long-range weak interactions. Addition of the full triplet-pairing component of $T_{2}$ to $\mathrm{CC} 0(+\mathrm{tDFT})$ is frequently better than adding just the parallel-spin correlation $(+\mathrm{pDFT})$, in agreement with theoretical arguments outlined in Section 2.2.

It is often said of traditional coupled cluster methods that they give "the right answer for the right reason." Yet, these approximations fail in the presence of strong correlation. Here, LC-CCD0+DFT, for example, can be considered as an attempt to avoid this failure while obtaining the right answer for the right reason via a different route: CCD0 correctly describes most of the opposite spin correlation without breaking down in the strong correlation regime; DFAs are most accurate in the 

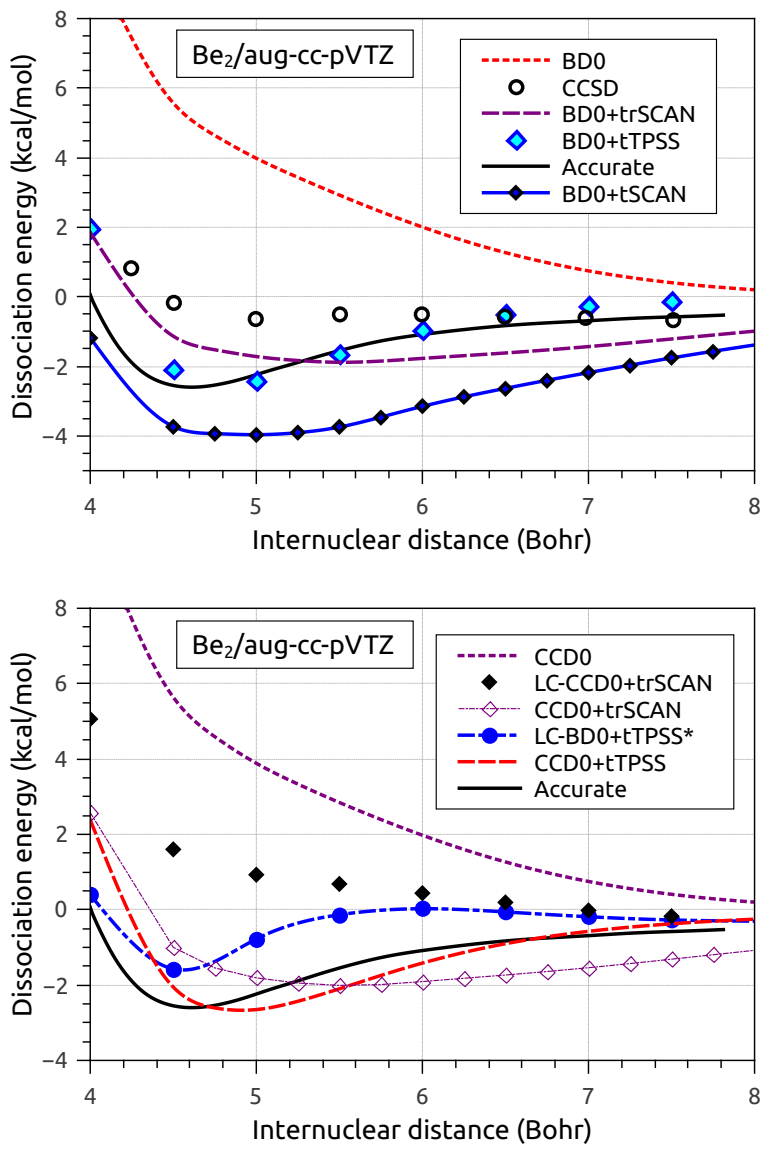

FIG. 5: Dissociation energy profiles for the Beryllium dimer calculated by various methods using an uncontracted Cartesian aug-cc-pVTZ basis. The accurate data are from explicitly correlated $r_{12}$ MRCI calculations from Ref. [135]. The CCSD data are from Ref. 134. The curve marked as LCBD0+tTPSS* uses the semiempirical prefactor of 1.5 on the dRPA correlation of Ref. 85.

short-range [137, while RPA is most accurate in the longrange [92 94]; and we add specifically the correlation absent in CCD0 from these last two. Nonetheless, there is room for improvement over the techniques presented here:

- The major drawback of CCD0 and BD0 is their $\mathcal{O}\left(N^{6}\right)$ scaling, which determines the cost of the combinations presented here. Additionally, the DFA correlation added to CCD0/BD0 is evaluated in terms of a multiple of the same-spin correlation. This has the disadvantage that resolutions for the parallel-spin correlation are imperfect, and also that same-spin correlation typically makes up for a larger fraction of the total correlation in the uniform electron gas as compared to molecules [30, 71, which may result in too low total energies in $\mathrm{CC} 0+\mathrm{DFT}$. Both of these problems can be fixed by appropriate combinations of DFT with pCCD, rather than BD0 and CCD0.
The scaling of pCCD is only $\mathcal{O}\left(N^{3}\right)$ (neglecting the basis transformation of the two-body interaction [11). Although pCCD misses more dynamic correlation than CCD0, the intrapair correlation is described almost exactly; pCCD closely reproduces seniority-zero FCI - an optimal linear combination of all configurations that preserve electron pairs [11] (i.e., all seniority zero configurations; see Ref. [138] for a detailed explanation of the concept of seniority). Hence, following the philosophy of the present work, we can add the correlation missing in pCCD by switching the discussion from the singlet/tripletpairing components of $\mathrm{CC} 0$ to intra/inter-pair correlation. Ways of extracting intra/inter-pair correlation from DFAs have been discussed by Savin et al. 139. Likewise, adding dRPA correlation to pCCD without double counting can be done with a similar strategy to the one done here by including only integrals that "break" electron pairs (seniority nonzero) in the $\mathbf{B}$ matrix of dRPA.

- Here, the DFA correlation is added to $\mathrm{CC} 0$ in a non-self-consistent manner. Although the effect of self-consistency is often small when adding dynamic DFA correlation to a wavefunction [72, it is possible that self-consistency can improve $\mathrm{CC} 0+\mathrm{DFT}$. In particular, it would be possible to make the singlet- and triplet-pairing components of $T_{2}$ to "talk" to each other-and without introducing the risk of breakdown in strongly correlated systemsby including an effective one-body potential from the DFA in core Hamiltonian of CC0.

- We have relied here on educated guesses for extracting the parallel spin and short-range correlation from existing functionals. Thus, our $\mathrm{CC} 0+\mathrm{DFT}$ methods can be refined by parametrizing a parallel spin correlation functional complementary to $\mathrm{CC} 0$, and doing a rigorous parametrization of said functional for the range separation. Related to this, LC-CC0+DFT may also benefit from a finer tuning of the range separation parameter.

Thus, it is likely that the already good results obtained here can be improved further, and that the cost of $\mathrm{CC} 0+\mathrm{DFT}$ be reduced by using pCCD $+\mathrm{DFT}$ combinations. Indeed, many other $\mathrm{CC} 0+\mathrm{DFT}$ (or $\mathrm{CC} 0+\mathrm{RPA}$ ) mixtures can be developed following the general strategies used here. Currently, we are working on some of these extensions and on testing $\mathrm{CC} 0+\mathrm{DFT}$ methods on larger systems; preliminary results have been encouraging. We hope that the ideas and results presented here can stimulate the further development of $\mathrm{CC} 0+\mathrm{DFT}$ methods.

\section{Acknowledgments}

This work was supported as part of the Center for the Computational Design of Functional Layered Materials, 
an Energy Frontier Research Center funded by the U.S. Department of Energy, Office of Science, Basic Energy
Sciences under Award \# de-sc0012575. GES is a Welch Foundation chair (C-0036).
[1] P.-D. Fan and P. Piecuch, Adv. Quantum Chem. 51, 1 (2006).

[2] I. W. Bulik, T. M. Henderson, and G. E. Scuseria, J. Chem. Theory and Comput. 11, 3171 (2015).

[3] G. E., Scuseria, T. M. Henderson, and D. C. Sorensen, J. Chem. Phys. 129, 231101 (2008).

[4] G. E. Scuseria, T. M. Henderson, and I. W. Bulik, J. Chem. Phys. 139, 104113 (2013).

[5] D. G. Peng, S. N. Steninman, H. van Aggelen, and W. T. Yang, J. Chem. Phys. 139, 104112 (2013).

[6] P. A. Limacher, P. W. Ayers, P. A. Johnson, S. de Baerdemacker, D. van Neck, and P. Bultinck, J. Chem. Theory Comput. 9, 1394 (2013).

[7] P. A. Limacher, T. D. Kim, P. W. Ayers, P. A. Johnson, S. de Baerdemacker, D. van Neck, and P. Bultinck, Mol. Phys. 112, 853 (2014).

[8] P. Tecmer, K. Boguslawski, P. A. Johnson, P. A. Limacher, M. Chan, T. Verstraelen, and P. W. Ayers, J. Phys. Chem. A 118, 9058 (2014).

[9] K. Boguslawski, P. Tecmer, P. W. Ayers, P. Bultinck, S. de Baerdemacker, and D. van Neck, Phys. Rev. B 89, 201106(R) (2014).

[10] T. Stein, T. M. Henderson, and G. E. Scuseria, J. Chem. Phys. 140, 214113 (2014)

[11] T. M. Henderson, I. W. Bulik, T. Stein, and G. E. Scuseria, J. Chem. Phys. 141, 244104 (2014).

[12] G. C. Lie and E. Clementi, J. Chem. Phys. 60, 1275 (1974).

[13] G. C. Lie and E. Clementi, J. Chem. Phys. 60, 1288 (1974).

[14] A. J. Pérez-Jiménez and J. M. Pérez-Jordá, Phys. Rev. A 75, 012503 (2007).

[15] R. Colle and O. Salvetti, Theor. Chim. Acta 52, 55 (1979).

[16] F. Moscardó and E. San-Fabián, Phys. Rev. A 44, 1549 (1991).

[17] E. Kraka, Chem. Phys. 161, 149 (1992).

[18] N. O. J. Malcolm and J. J. W. McDouall, Phys. Chem. 100, 10131 (1996)

[19] N. O. J. Malcolm and J. J. W. McDouall, Phys. Chem. A 101, 8119 (1997).

[20] T. Leininger, H. Stoll, H. Werner, and A. Savin, Chem. Phys. Lett. 275, 151 (1997).

[21] R. Pollet, A. Savin, T. Leininger, and H. Stoll, J. Chem. Phys. 116, 1250 (2002).

[22] A. Stoyanova, A. M. Teale, J. Tolouse, T. Helgaker, and E. Fromager, J. Chem. Phys. 139, 134113 (2013).

[23] E. D. Hedegård, S. Knecht, J. S. Kielberg, H. J. Aa. Jensen, and M. Reiher, J. Chem. Phys. 142, 224108 (2015).

[24] Y. Cornaton and E. Fromager, Int. J. Quantum Chem. 114, 1199 (2014).

[25] A. J. Garza, T. M. Henderson, I. W. Bulik, and G. E. Scuseria, Phys. Chem. Chem. Phys. 17, 22412 (2015).

[26] W. Wu and S. Shaik, Chem. Phys. Lett. 301, 37 (1999).

[27] S. Grimme and M. Waletzke, J. Chem. Phys. 111, 5645 (1999).
[28] B. Miehlich, H. Stoll, and A. Savin, Mol. Phys. 91, 527 (1997).

[29] J. Gräfenstein and D. Cremer, Chem. Phys. Lett. 316, 569 (2000).22

[30] J. Gräfenstein and D. Cremer, Mol. Phys. 103, 279 (2005).

[31] C. Gutlé and A. Savin, Phys. Rev. A 75, 032519 (2007).

[32] C. Gutlé, A. Savin, J. B. Krieger, J. Chen, Int. J. Quantum Chem. 75, 885 (1999).

[33] H. Stoll, Chem. Phys. Lett. 376, 141 (2003).

[34] R. Takeda, S. Yamanaka, and K. Yamaguchi, Chem. Phys. Lett. 366, 321 (2002).

[35] R. Takeda, S. Yamanaka, and K. Yamaguchi, Int. J. Quantum Chem. 96, 463 (2004).

[36] S. Gusarov, P. Malmqvist, and R. Lindh, Mol. Phys. 102, 2207 (2004).

[37] S. Gusarov, P. Malmqvist, R. Lindh, and B. O. Roos, Theor. Chem. Acc. 112, 84 (2004).

[38] T. Tsuchimochi, G. E. Scuseria, and A. Savin, J. Chem. Phys. 132, 024111 (2010).

[39] K. Sharkas, A. Savin, H. J. Aa. Jensen, and J. Tolouse, J. Chem. Phys. 137, 044104 (2012).

[40] G. Li Manni, R. K. Carlson, S. Luo, D. Ma, J. Olsen, D. G. Truhlar, and L. Gagliardi, J. Chem. Theory Comput. 10, 3669 (2014).

[41] A. J. Garza, T. M. Henderson, I. W. Bulik, and G. E. Scuseria, J. Chem. Phys. 142, 044109 (2015).

[42] A. J. Garza, C. A. Jiménez-Hoyos, and G. E. Scuseria, J. Chem. Phys. 138, 134102 (2013).

[43] A. J. Garza, C. A. Jiménez-Hoyos, and G. E. Scuseria, J. Chem. Phys. 140, 244102 (2014).

[44] J. D. Goodpaster, T. A. Barnes, F. R. Manby, and T. F. Miller III, J. Chem. Phys. 140 18A507 (2014).

[45] E. Fromager, Mol. Phys. 113, 419 (2015).

[46] G. Knizia and G. K. L. Chan, Phys. Rev. Lett. 109, 186404 (2012).

[47] I. W. Bulik, G. E. Scuseria, and J. Dukelsky, Phys. Rev. B 89, 035140 (2014)

[48] J. P. Perdew, A. Savin, and K. Burke, Phys. Rev. A 51, 4531 (1995).

[49] A. J. Cohen, P. Mori-Sánchez, and W. Yang, Science 321, 792 (2008).

[50] T. Tsuchimochi and G. E. Scuseria, J. Chem. Phys. 131, 121102 (2009).

[51] C. A. Jiménez-Hoyos, T. M. Henderson, T. Tsuchimochi, and G. E. Scuseria, J. Chem. Phys. 136, 164109 (2012).

[52] S. R. White, Phys. Rev. Lett. 69, 2863 (1992).

[53] T. M. Henderson, I. W. Bulik, and G. E. Scuseria, J. Chem. Phys. 142, 214116 (2015).

[54] C. A. Jiménez-Hoyos, R. R. Rodríguez-Guzmán, and G. E. Scuseria, J. Chem. Phys. 139, 204102 (2013).

[55] T. Tsuchimochi and T. Van Voorhis, J. Chem. Phys. 141, 164117 (2014).

[56] P. Limacher, P. Ayers, P. Johnson, S. De Baerdemacker, D. Van Neck, and P. Bultinck, Phys. Chem. Chem. Phys. 16, 5061 (2014). 
[57] P. Jeszenszki, P. R. Nagy, T. Zoboki, Á. Szabados, and P. R. Surján, Int. J. Quantum Chem. 114, 1048 (2014).

[58] K. Pernal, J. Chem. Theory Comput. 10, 4332 (2014).

[59] E. Pastorczak and K. Pernal, Phys. Chem. Chem. Phys. 17, 8622 (2015).

[60] P. A. Limacher, J. Chem. Theory Comput. 11, 3629 (2015).

[61] K. Boguslawski and P. W. Ayers, arXiv:1508.02782 [physics.chem-ph] (2015).

[62] J. Geertsen and J. Oddershede, J. Chem. Phys. 85, 2112 (1986).

[63] G. E. Scuseria, C. L. Janssen, and H. F. Schaefer III, J. Chem. Phys. 89, 7382 (1988).

[64] P. Piecuch, and J. Paldus, J. Theor. Chimica Acta 78, 65 (1990).

[65] C. E. Dykstra, Chem. Phys. Lett. 45, 466 (1977).

[66] N. C. Handy, J. A. Pople, M. Head-Gordon, K. Raghavachari, and G. W. Trucks, Chem. Phys. Lett. 164, 185 (1989).

[67] R. Kobayashi, N. C. Handy, R. D. Amos, G. W. Trucks, M. J. Frisch, and J. A. Pople, J. Chem. Phys. 95, 6723 (1991).

[68] C. D. Sherrill, A. I. Krylov, E. F. C. Byrd, and M. Head-Gordon, J. Chem. Phys. 109, 4171 (1998).

[69] G. E. Scuseria and H. F. Schaefer, Chem. Phys. Lett. 142, 354 (1987).

[70] D. G. Liakos and F. Neese, J. Chem. Theory Comput. DOI: 10.1021/acs.jctc.5b00359 (2015).

[71] H. Stoll, C. E. Pavlidou, and H. Preuss, Theor. Chim. Acta 49, 143 (1978).

[72] H. Stoll and A. Savin, Density Functional Theory Methods in Physics, edited by R. M. Dreizler and J. da Providencia (Plenum, New York, 1985), p. 177.

[73] J. Tao, J. P. Perdew, V. N. Staroverov, and G. E. Scuseria, Phys. Rev. Lett. 91, 146401 (2003).

[74] J. Sun, A. Ruzsinszky, and J. P. Perdew, Phys. Rev. Lett. 115, 036402 (2015).

[75] P. Gori-Giorgi and J. P. Perdew, Phys. Rev. B 69, 041103(R) (2004).

[76] P. Gori-Giorgi, F. Sacchetti, and G. B. Bachelet, Phys. Rev. B 61, 7353; 66, 159901(E) (2002).

[77] J. Tao, J. P. Perdew, and A. Ruzsinszky, Proc. Natl. Acad. Sci. U.S.A. 109, 18 (2012).

[78] J. Klimeš and A. Michaelides, J. Chem. Phys. 137, 120901 (2012).

[79] A. Savin, and H. Flad, Int. J. Quantum Chem. 56, 327 (1995).

[80] H. Iikura, T. Tsuneda, T. Yanai, and K. Hirao, J. Chem. Phys. 115, 3540 (2001).

[81] T. Yanai, D. P. Tew, and N. C. Handy, Chem. Phys. Lett. 393, 51 (2004).

[82] O. A. Vydrov, J. Heyd, A. V. Krukau, and G. E. Scuseria, J. Chem. Phys. 125, 074106 (2006).

[83] O. A. Vydrov and G. E. Scuseria, J. Chem. Phys. 125, 234109 (2006).

[84] B. G. Janesko, T. M. Henderson, and G. E. Scuseria, Phys. Chem. Chem. Phys. 11, 443 (2009).

[85] B. G. Janesko, T. M. Henderson, and G. E. Scuseria, J. Chem. Phys. 130, 081105 (2009).

[86] H. Eshuis, J. E. Bates, and F. Furche, Theor. Chem. Acc. 131, 1084 (2012).

[87] J. Toulouse, W. Zhu, A. Savin, G. Jansen, and J. C. Ángyán, J. Chem. Phys. 135084119 (2011).
[88] R. Seeger and J. A. Pople, J. Chem. Phys. 66, 3045 (1977).

[89] F. Furche, Phys. Rev. B 64, 195120 (2001).

[90] Y. Cui, I. W. Bulik, C. A. Jiménez-Hoyos, T. M. Henderson, and G. E. Scuseria, J. Chem. Phys. 139, 154107 (2013).

[91] F. Furche, J. Chem. Phys. 129, 114105 (2008).

[92] J. C. Ángyán, I. C. Gerber, and J. Tolouse, Phys. Rev. A 72, 012510 (2005).

[93] J. F. Dobson, A. White, and A. Rubio, Phys. Rev. Lett. 96, 073201 (2006).

[94] Z. Yan, J. P. Perdew, and S. Kurth, Phys. Rev. B 61, 16430 (2000).

[95] S. Paziani, S. Moroni, P. Gori-Giorgi, and G. B. Bachelet, Phys. Rev. B 73, 155111 (2006).

[96] T. Stein, L. Kronik, and R. Baer, J Am. Chem. Soc. 131, 2818 (2009).

[97] S. Refaely-Abramson, R. Baer, and L. Kronik, Phys. Rev. B 84, 075144 (2011).

[98] L. Kronik, T. Stein, S. Refaely-Abramson, and R. Baer, J. Chem. Theory Comput. 8, 1515 (2012).

[99] J. Autschbach and M. Srebro, Acc. Chem. Res. 47, 2592 (2014).

[100] A. J. Garza, N. A. Wazzan, A. M. Asiri, and G. E. Scuseria, J. Phys. Chem A 118, 11787 (2014).

[101] E. Fromager, J. Tolouse, and H. J. Aa. Jensen, J. Chem. Phys. 126, 074111 (2007).

[102] E. Fromager, F. Réal, P. Wålin, U. Wahlgren, and H. J. Aa. Jensen, J. Chem. Phys. 131, 054107 (2009).

[103] A. Karolewski, L. Kronik, and S. Kümmel, J. Chem. Phys. 138, 204115 (2013).

[104] A. V. Krukau, G. E. Scuseria, J. P. Perdew, and A. Savin, J. Chem. Phys. 129, 124103 (2008).

[105] T. M. Henderson, B. G. Janesko, G. E. Scuseria, and A. Savin, Int. J. Quantum Chem. 109, 2023 (2009).

[106] M. Dion, H. Rydberg, E. Schröder, D. C. Langreth, and B. I. Lundqvist, Phys. Rev. Lett. 92, 246401 (2004).

[107] K. Lee, E. D. Murray, L. Kong, B. I. Lundqvist, and D. C. Langreth, Phys. Rev. B 82, 081101(R) (2010).

[108] O. A. Vydrov and T. Van Voorhis, J. Chem. Phys. 130, 104105 (2009).

[109] T. Sato and H. Nakai, J. Chem. Phys. 131, 224104 (2009).

[110] M. J. Frisch, G. W. Trucks, H. B. Schegel et al., GausSIAN Development Version, Revision H.21, Gaussian Inc., Wallingford, CT, 2009.

[111] A. J. Pérez-Jiménez, J. M. Pérez-Jordá, and J. C. Sancho-García, J. Chem. Phys. 127, 104102 (2007).

[112] P.J. Linstrom and W.G. Mallard, Eds., NIST Chemistry WebBook, NIST Standard Reference Database Number 69, National Institute of Standards and Technology, Gaithersburg MD, 20899, http://webbook.nist.gov, (retrieved August 25, 2015).

[113] NIST Computational Chemistry Comparison and Benchmark Database, NIST Standard Reference Database Number 101 Release 16a, August 2013, Editor: Russell D. Johnson III http://cccbdb.nist.gov/ (retrieved August 25, 2015).

[114] G. E. Scuseria, Int. J. Quantum Chem. 55, 165 (1995).

[115] I. Lindgren and S. Salomonson, Int, J. Quantum Chem. 90, 294 (2002).

[116] Y. Zhao and D. G. Truhlar, Theor. Chem. Acc. 120, 215 (2008). 
[117] V. N. Staroverov, G. E. Scuseria, J. Tao, and J. P. Perdew, J. Chem. Phys. 119, 12129 (2003).

[118] Y. Zhao and D. G. Truhlar, J. Phys. Chem. A 110, 10478 (2006).

[119] J. P. Perdew, S. Kurth, A. Zupan, and P. Blaha, Phys. Rev. Lett. 82, 2544 (1999); 82, 5179(E) (1999).

[120] C. Lee, W. Yang, and R. G. Parr, Phys. Rev. B 37, 785 (1988).

[121] M. Filatov and D. Cremer, J. Chem. Phys. 123, 124101 (2005).

[122] K. T. Tang and J. P. Toennies, J. Chem. Phys. 118, 4976 (2003).

[123] Y. Zhao and D. G. Truhlar, J. Phys. Chem. A 109, 5656 (2005).

[124] J. Tao, J. P. Perdew, and A. Ruzsinszky, Phys. Rev. B 81, 233102 (2010).

[125] J. Sun, B. Xiao, R. Haunschild, P. Hao, A. Ruzsinszky, G. I. Csonka, G. E. Scuseria, and J. P. Perdew, Phys. Rev. Lett. 111, 106401 (2013).

[126] A. Dutta and C. D. Sherrill, J. Chem. Phys. 118, 1610 (2003).

[127] J. Paldus, P. Piecuch, L. Pylypow, and B. Jeziorski, Phys. Rev. A 47, 2738 (1993).

[128] K. Kowalski and K. Jankowski, Chem. Phys. Lett. 290, 180 (1998).

[129] K. Kowalski and K. Jankowski, Phys. Rev. Lett. 81,
1195 (1998).

[130] K. Jankowski and K. Kowalski, J. Chem. Phys. 111, 2952 (1999).

[131] T. Van Voorhis and M. Head-Gordon, J. Chem. Phys. 113, 8873 (2000).

[132] G. A. Petersson and S. L. Licht, J. Chem. Phys. 75, 4556 (1981).

[133] M. El Khatib, G. L. Bendazzoli, S. Evangelisti, W. Helal, T. Leininger, L. Tenti, and C. Angeli, J Phys. Chem. A 118, 6664 (2014).

[134] V. F. Lotrich, R. J. Bartlett, and I. Grabowski, Chem. Phys. Lett. 405, 43 (2005).

[135] R. J. Gdanitz, Chem. Phys. Lett. 312, 578 (1999).

[136] C. Sosa, J. Noga, and R. J. Bartlett, J. Chem. Phys. 88, 5974 (1988).

[137] J. Toulouse, F. Colonna, and A. Savin, Phys. Rev. A 70, 062505 (2004).

[138] L. Bytautas, T. M. Henderson, C. A. Jiménez-Hoyos, J. K. Ellis, and G. E. Scuseria, J. Chem. Phys. 135, 044119 (2011).

[139] Pair correlation energies and local spin-density functionals, A. Savin, H. Stoll, H. Preuss, dans: J. P. Dahl, J. Avery, eds., Local density approximations in quantum chemistry and solid state physics, Plenum, New York, 1984, p. 263. 